\title{
3-D shape perception
}

\author{
ZYGMUNT PIZLO and MONIKA SALACH-GOLYSKA \\ Purdue University, West Lafayette, Indiana
}

\begin{abstract}
In this paper, we analyze and test three theories of 3-D shape perception: (1) Helmholtzian theory, which assumes that perception of the shape of an object involves reconstructing Euclidean structure of the object (up to size scaling) from the object's retinal image after taking into account the object's orientation relative to the observer, (2) Gibsonian theory, which assumes that shape perception involves invariants (projective or affine) computed directly from the object's retinal image, and (3) perspective invariants theory, which assumes that shape perception involves a new kind of invariants of perspective transformation. Predictions of these three theories were tested in four experiments. In the first experiment, we showed that reliable discrimination between a perspective and nonperspective image of a random polygon is possible even when information only about the contour of the image is present. In the second experiment, we showed that discrimination performance did not benefit from the presence of a textured surface, providing information about the 3-D orientation of the polygon, and that the subjects could not reliably discriminate between the 3-D orientation of the textured surface and that of a shape. In the third experiment, we compared discrimination for solid shapes that either had flat contours (cuboids) or did not have visible flat contours (cylinders). The discrimination was very reliable in the case of cuboids but not in the case of cylinders. In the fourth experiment, we tested the effectiveness of planar motion in perception of distances and showed that the discrimination threshold was large and similar to thresholds when other cues to 3-D orientation were used. All these results support perspective invariants as a model of 3-D shape perception.
\end{abstract}

Shape is defined in this paper conventionally as the property of the contour of a figure or of the surface of an object that does not change under translation, rotation, and size scaling (i.e., under similarity transformations). Perception of shapes of objects involves making inferences about the three-dimensional (3-D) shape of an object on the basis of its 2-D retinal image (or several images). This poses a problem, because the shape of the retinal image depends not only on the shape of the object but also on the orientation of the object relative to the observer. However, the percept of the shape of a given object tends to be constant despite changes in the shape of the object's retinal image. This phenomenon is called shape constancy. Consider an example. Figure 1 shows an object from two different viewing directions $45^{\circ}$ apart. If the reader perceives these two different images as representing the same shape, shape constancy has been achieved.

There are several theories of shape constancy (see Pizlo, 1994, for a recent review of prior theories and experiments on shape constancy). In this paper, we will concentrate on two traditional theories (Helmholtzian and Gibsonian) and also on the perspective invariants theory formulated recently by Pizlo (1994). According to Helmholtzian theory, shape constancy involves reconstructing a 3-D Euclidean structure of the object up to size scaling (i.e., the similarity structure) from the retinal image after taking into account the

The authors are grateful to Myron Braunstein, Joseph Lappin, James Todd, and an anonymous reviewer for their helpful suggestions and comments. Correspondence should be addressed to Z. Pizlo, Purdue University, 1364 Psychological Sciences Bldg., West Lafayette, IN 479071364 (e-mail: pizlo@psych.purdue.edu). object's orientation relative to the observer (e.g., Marr, 1982; Thouless, 1931). The 3-D orientation can be computed from such cues as, for example, texture, shading, motion, and linear perspective. If this reconstruction is always performed correctly, the perceived shape will be the same regardless of the orientation of the object relative to the observer.

According to Gibsonian theory, shape constancy is not based on taking the object's orientation into account. Instead, shape constancy involves projective invariants (Cassirer, 1938/1944; Courant \& Robbins, 1941; Gibson, 1950; Mundy \& Zisserman, 1992). Projective invariants are geometrical properties of a shape that are preserved in any perspective or projective image of the shape. If the range of the object in depth is small relative to the distance of the object from the observer, projective transformation reduces to affine, and, thus, affine invariants can be used instead of projective invariants (Mundy \& Zisserman, 1992).

In the past, Helmholtzian theory has been commonly accepted as an adequate explanation of shape constancy. There are, however, problems with this theory-namely, from a mathematical point of view, reconstruction of similarity properties of a 3-D object from its 2-D image, after taking cues to 3-D orientation into account, is an ill-posed problem. That is, a given 2-D image determines more than one 3-D interpretation (in fact, it determines an infinite number of interpretations). To obtain a unique interpretation, it is necessary to use some additional assumptions (e.g., that the surface is Lambertian, that texture elements are known or, at least, regular [constant size, constant shape]). These assumptions are often satisfied, at least approximately, so it is possible to obtain a unique 3-D interpretation. However, this interpretation is mathematically 

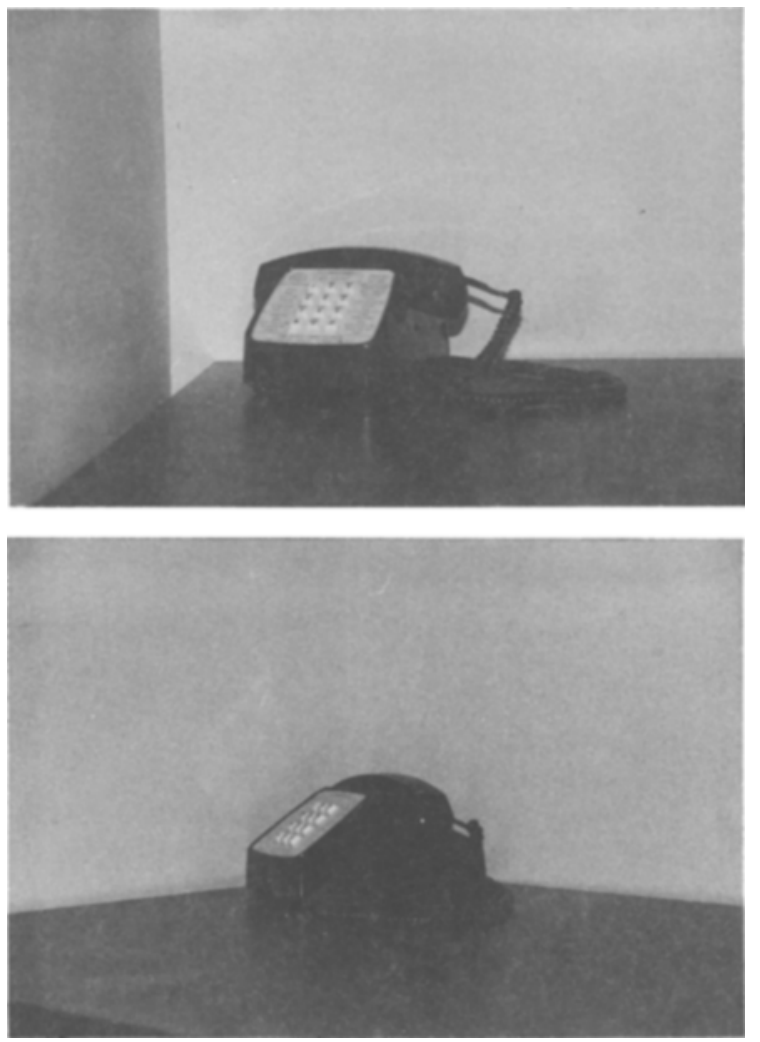

Figure 1. Two images of an object obtained from viewing directions $45^{\circ}$ apart. It is easy to achieve shape constancy in the case of this object.

unstable. That is, a small change in the image gives rise to large changes in the 3-D interpretation. This is a serious problem because, in the presence of noise or occlusion, which is a rule rather than an exception in human and computer vision, the interpretation may be very inaccurate. There have been some attempts to elaborate the computational methods of reconstruction, such as Bajcsy's (1985) active vision or Poggio, Torre, and Koch's (1985) regularization method, in order to obtain more stable solutions. However, these elaborations do not improve the reliability and accuracy of reconstruction significantly. As a result, there is currently no theory of vision (human or computer) that can specify how to solve the reconstruction problem accurately and reliably for an arbitrary 3-D object.

The mathematical considerations presented above, which suggest that reconstructing the 3-D similarity structure of an object should be a difficult problem, receive some support from results of psychophysical experiments. Namely, it was shown that the judgments of a human observer about distances or angles in 3-D are unreliable and inaccurate (Todd \& Bressan, 1990; Todd \& Norman, 1991, 1994; Todd \& Reichel, 1989). These results imply either that perception of shape must always be unreliable and inaccurate because reconstruction of its Euclidean or similarity properties is poor or that reliable and accurate perception of shape is not based on reconstructing the 3-D structure of an object. Recent experimental tests of shape constancy seemingly provide support for the former possibility. By testing human recognition of 3-D wire objects or star objects in the presence of such cues as binocular disparity, vergence, accommodation, shading, texture, and motion, which could be used to compute the 3-D orientation and distance of the object relative to the observer, it has been shown that shape constancy is very difficult or even impossible to achieve (Edelman \& Bulthoff, 1992; Rock \& DiVita, 1987; Rock, Wheeler, \& Tudor, 1989). Figure 2 illustrates this result. The figure shows a crumpled piece of paper seen from two different directions $45^{\circ}$ apart. Despite the presence of cues to 3-D orientation (texture and shading), it is very difficult, if possible at all, to recognize that these two images were produced by the same object. This fact represents the failure of shape constancy.

Note, however, that the 3-D wire objects, star objects, and crumpled pieces of paper either are not likely to be encountered in our everyday life or are unimportant. Therefore, the results from these studies may not (and, it will be shown, do not) generalize to other objects. For example, Stavrianos (1945) and Pizlo (1994) showed that simple 2-D shapes (quadrilaterals) are perceived accurately and reliably from their perspective images even when the cues to 3-D orientation are greatly reduced, which means that shape constancy is possible for such shapes and that it is
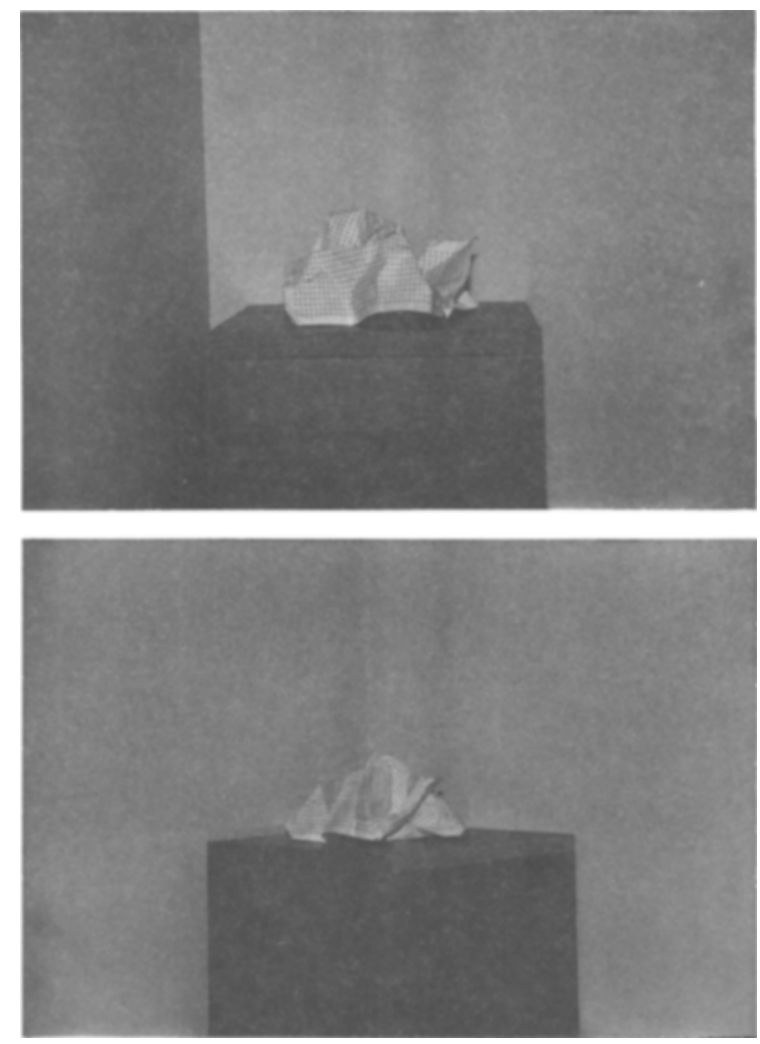

Figure 2. Two images of an object obtained from viewing directions $45^{\circ}$ apart. It is difficult to achieve shape constancy in the case of this object. 
not likely to be based on reconstructing Euclidean or similarity structure of the shapes by taking their orientation into account.

Achieving shape constancy does not seem to be limited to the case of simple 2-D shapes, such as those studied by Stavrianos and Pizlo, because, as can be seen in Figure 1, it is rather easy to recognize that the two different images represent one and the same object. One possible, but invalid argument would be that the object in Figure 1 is familiar, and, therefore, that familiarity allows its recognition from any viewing direction. It is easy to show, however, that familiarity is not necessary for achieving shape constancy. Consider the two different images in Figure 3. They represent a parallelepiped seen from viewing directions $60^{\circ}$ apart. This object is not likely to be familiar to the reader. Moreover, these images do not provide cues that would be sufficient to reconstruct its 3-D similarity structure. Nevertheless, it is probably easy for the reader to recognize that these two different images represent one and the same 3-D object. We want to point out, however, that some cues to 3-D orientation that could be used to reconstruct the 3-D structure are present in these images. For example, the compression of the faces in the image can be used to estimate their tilts and slants (Witkin,

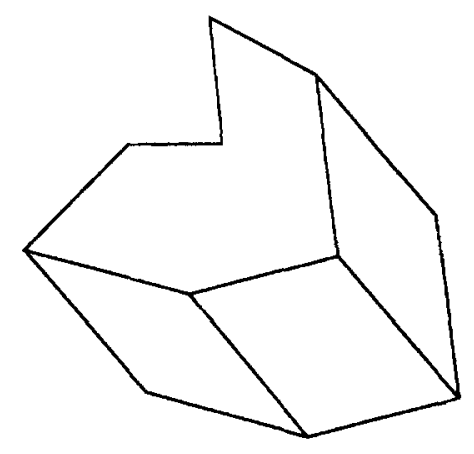

a

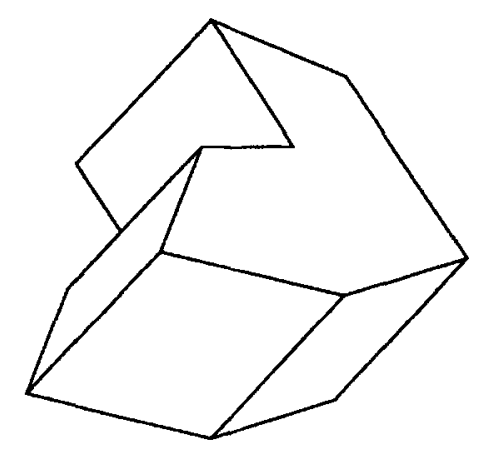

b

Figure 3. Two images of a parallelepiped obtained from viewing directions $60^{\circ}$ apart. It is easy to achieve shape constancy in the case of this object.
1981). ${ }^{1}$ This estimate, however, is based on a strong and unlikely assumption that the contours of the faces have circular symmetry. If this assumption is not satisfied, the 3-D orientation cannot be computed accurately and, as a result, the 3-D similarity structure cannot be reconstructed accurately.

The fact that shape constancy is possible for some 3-D objects has been recently demonstrated in psychophysical experiments. Johnston, Cumming, and Landy (1994) have shown that the depth-to-height ratio of a rotating cylinder viewed binocularly can be judged accurately by subjects. Similarly, Durgin, Proffitt, Olson, and Reinke (in press) have shown that the height-to-base ratio of a circular cone viewed binocularly can be judged accurately.

The example in Figure 3, together with results of prior experiments, shows that if shape perception is studied with objects other than 3-D wires, star objects, or crumpled pieces of paper, shape constancy can be achieved reliably and accurately for 2-D and 3-D shapes even though reconstruction of the 3-D Euclidean or similarity structure of objects is not accurate or reliable. These results seem to be consistent with Gibsonian theory, which assumes that shape constancy is based on projective or affine invariants. However, Pizlo (1994) has shown that affine and projective invariants-the only conventional invariants that can be applied in the case of a single perspective image-cannot account for human shape perception. Consider Figure 4, which shows an image of a parallelepiped different from the one shown in Figure 3. The parallelepiped in Figure 4 was obtained by stretching the parallelepiped in Figure 3 along the direction orthogonal to its bases. This means that the object shown in Figure 4 is an affine transformation of the object shown in Figure 3, which implies that in affine and projective geometries these two objects are equivalent and that affine and projective invariants cannot discriminate between these two objects. However, these objects do not look the same and, in fact, the images in Figures 3 and 4 could not be produced by one and the same object. This observation contradicts the theory of shape constancy based on affine or projective invariants.

To account for existing results on shape constancy, Pizlo (1994) has proposed a theory based on perspective invariants that were formulated by Pizlo and Rosenfeld (1992). Perspective invariants seem to be similar to projective or affine invariants. However, there are some essential differences between perspective invariants and conventional invariants (e.g., projective or affine). For example, conventional invariants require that the transformations of interest form a group, but perspective transformations do not form a group because a composition of two or more perspectivities is a projectivity, not a perspectivity (Springer, 1964). In fact, image formation is not even equivalent to a general perspectivity, but only to some subclass of perspectivities.

The next section will describe the main aspects of the perspective invariants theory of shape constancy (Pizlo, 1994), including the geometrical properties of image formation, invariants of image formation (i.e., perspective in- 


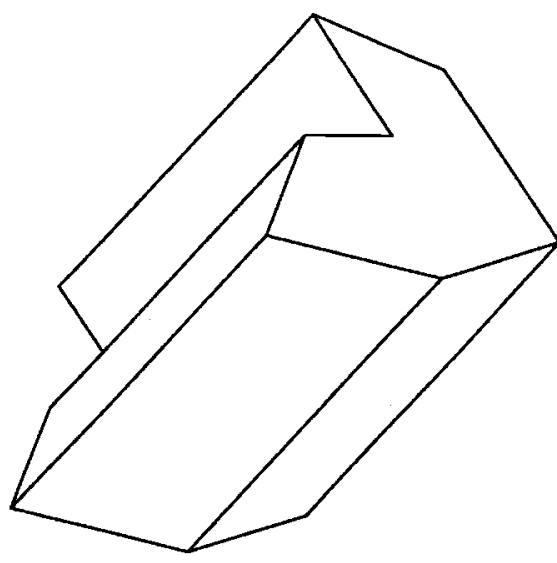

Figure 4. An image of a parallelepiped, which was obtained from the parallelepiped shown in Figure 3 by stretching. It is easy to see that Figures 3 and 4 represent different objects.

variants) formulated by Pizlo and Rosenfeld (1992), and perceptual implications of perspective invariants.

\section{PERSPECTIVE INVARIANTS THEORY OF SHAPE CONSTANCY}

\section{Image Formation}

Consider the relation between the 3-D scene and its retinal image. This relation is represented by a many-to-one mapping, since many points in the scene can give rise to one point in the retina. Because many-to-one mapping does not allow for a unique interpretation of a 3-D scene on the basis of its 2-D image, one has to either use more images of the same 3-D scene (as is the case when a stationary observer is viewing the scene with two eyes or when there is a relative motion between the scene and the observer) or assume that the objects out there are flat or have flat faces. In the case of a flat object, image formation represents a mapping from a plane to the retina, and this mapping is one-to-one. For simplicity of mathematical derivation, it can be assumed that the retina itself is flat, too. In fact, a planar approximation to the (spherical) retina is quite accurate in the central part of the retina, where eccentricities are less than about $20^{\circ}$. This part of the retina, in which the visual acuity is the highest, is usually involved in shape and pattern perception.

Perspective invariants have been formulated by Pizlo and Rosenfeld (1992) for the case of a flat object and its single retinal image. Image formation, in such a case, is called perspective transformation between two planes. Note, however, that in a general perspectivity the center of projection is not fixed and it can have an arbitrary position relative to the image plane. In the eye, however, the center of projection (i.e., the nodal point) is fixed relative to the image plane (retina). This means that image formation in the eye is a special case perspectivity (see Pizlo, 1993, 1994, and Pizlo, Rosenfeld, \& Weiss, in press-a, in press-b, for more details about the geometry of image formation).
This special case has only 3 free parameters that represent 3 degrees of freedom of the object plane: slant, tilt, and distance from the nodal point. Note that the other 3 parameters of the object plane representing translation and rotation on the object plane are not free in perspective in general and in image formation in particular. Perspective mapping between planes involves straight lines (light rays); therefore, the nodal point, the object point, and the corresponding image point must be collinear. It is clear that no translation or rotation on the object (or image) is allowed because this would require bending the light rays!

Let $C$ be the distance between the nodal point and the object plane measured along the line orthogonal to the image plane. Let $f$ be the distance of the nodal point from the image plane. Let $\sigma$ be slant and $\tau$ be tilt of the object plane. Following the notation used by Pizlo and Rosenfeld $(1992)$, where $\left(x^{\prime}, y^{\prime}\right)$ is a point in the object plane and $(x, y)$ is its image, the perspective relationship in the eye is represented by the following equations:

$x=f \cdot\left(x^{\prime} \cdot \cos \sigma \cdot \cos \tau-y^{\prime} \cdot \sin \tau\right) /\left(x^{\prime} \cdot \sin \sigma+C\right)$

$y=f \cdot\left(x^{\prime} \cdot \cos \sigma \cdot \sin \tau+y^{\prime} \cdot \cos \tau\right) /\left(x^{\prime} \cdot \sin \sigma+C\right)$. (1)

After solving Equations 1 for $x^{\prime}$ and $y^{\prime}$, one obtains equations for inverse perspective relationship in the eye:

$$
\begin{aligned}
x^{\prime}= & C \cdot(x \cdot \cos \tau+y \cdot \sin \tau) / \\
& (\cos \sigma \cdot(f-x \cdot \tan \sigma \cdot \cos \tau-y \cdot \tan \sigma \cdot \sin \tau)) \\
y^{\prime}= & C \cdot(-x \cdot \sin \tau+y \cdot \cos \tau) / \\
& (f-x \cdot \tan \sigma \cdot \cos \tau-y \cdot \tan \sigma \cdot \sin \tau) .
\end{aligned}
$$

Which equations should be used to derive invariants: equations representing perspectivity (Equations 1) or inverse perspectivity (Equations 2)? If perspectivity in general and image formation in particular were a group of transformations (and they are not), using either Equations 1 or Equations 2 would produce the same invariants, simply because, in a group of transformations, an inverse transformation is also a transformation from this group. Image formation, however, is not a group of transformations, because two group axioms are not satisfied: a composition of two perspectivities in general and two image formations in particular is not a perspectivity. Instead, it is a projectivity (i.e., the closure axiom is not satisfied). Next, inverse image formation is not an image formation, simply because, in the eye, images are formed on the retina and not on the object plane. ${ }^{2}$ The violation of these two group axioms has quite important implications. First, the fact that inverse image formation is not an image formation implies that using Equations 1 can produce invariants different from those produced using Equations 2. Second, the fact that closure axiom is not satisfied in image formation implies that image formation does not have invariants! An invariant of a transformation, according to a conventional definition, is an intrinsic property of a given image, and the value of this invariant remains the same in any transformation of this image. Assume that image formation has a conventional invariant $P$, which is 
invariant only under image formation and not under more general transformation (i.e., projective). Take an image $I_{1}$ and compute this invariant $P\left(I_{1}\right)$. Then, transform $I_{1}$ to $I_{2}$, by applying an image formation. Next, compute the invariant $P\left(I_{2}\right)$. Clearly, $P\left(I_{2}\right)=P\left(I_{1}\right)$, because $P$, as an invariant, must preserve its value. Next, transform $I_{2}$ to $I_{3}$, by applying an image formation and compute the invariant $P\left(I_{3}\right)$. Again, $P\left(I_{3}\right)=P\left(I_{2}\right)$, because $P$ must preserve its value. But, this means that $P\left(I_{3}\right)=P\left(I_{1}\right)$. However, $I_{3}$ is a result of a composition of two image formations, and this composition is not an image formation, but it is a projectivity. Thus, if an image formation has an invariant, it is a projective invariant, rather than an invariant of image formation.

This analysis, which shows that a transformation that is not a group does not have invariants, explains perhaps why mathematicians were not interested in perspectivity in general and image formation in particular. It has been commonly accepted in the past that an adequate model for vision is a projective geometry, because it is the least general geometry that has all properties of perspective (Cassirer, 1938/1944; Courant \& Robbins, 1941; Gibson, 1950; Johansson, 1977). The problem with projective geometry is that it is too general. Projectivity between two planes involves eight free parameters, whereas image formation involves only three. As a result, there are projective transformations that are never observed in the process of image formation and that are not equivalent perceptually. For example, in projective geometry, all convex quadrilaterals are equivalent, which means that if perception was based on projective invariants, all convex quadrilaterals would be perceived as having the same shape! But, this is not the case. Figure 5 shows another example illustrating the difference between projective and perspective. Figure 5a shows a polygon, Figure $5 \mathrm{~b}$ shows its perspective image, and Figure $5 \mathrm{c}$ shows its projective image. It is probably clear to the reader that Figures 5a and $5 \mathrm{~b}$ look like the same shape differently oriented in 3-D, but Figures $5 \mathrm{a}$ and $5 \mathrm{c}$ do not look like the same shape. However, if shape constancy was based on projective invariants, all three polygons would be perceptually equivalent (see Pizlo, 1994, and Pizlo et al., in press-a, in press-b, for other examples of projectively equivalent shapes that are not equivalent perceptually). Therefore, to explain the perception of shape one needs perspective, rather than projective invariants. However, such perspective invariants cannot be intrinsic properties of a given shape or pattern. Instead, the invariants must involve both the object and its image, and they should allow determining whether the two patterns are equivalent under image formation. In other words, using perspective invariants would allow establishing equivalence under transformations that are not groups. Despite the fact that such equivalence of shapes under perspective or image formation was not studied in mathematics, it seems to be more relevant for vision applications than invariants of conventional transformations. ${ }^{3}$

The discussion presented in the last two paragraphs implies that Equations 1 and 2 may lead to different invariants (or different shape equivalences). Pizlo and Rosen-

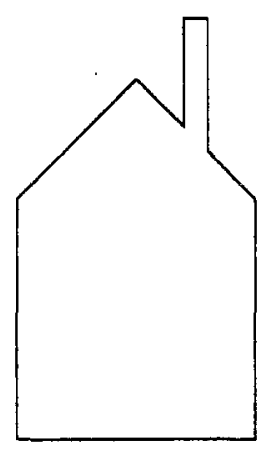

a

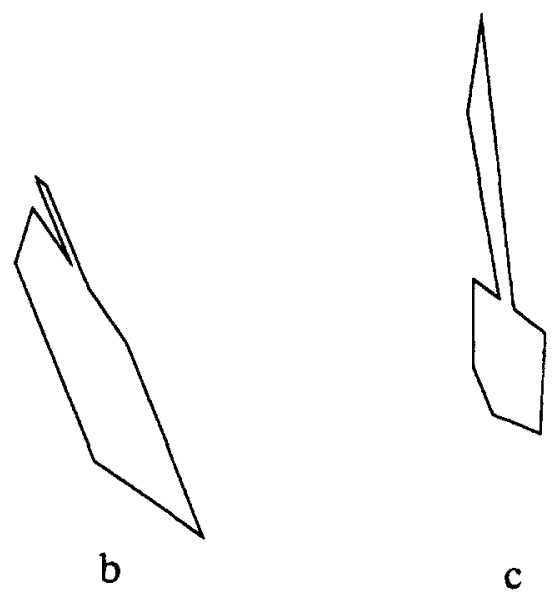

Figure 5. Illustration of perspective and projective transformations. (a) A frontal view of a polygon. (b) A perspective transformation of a with slant $75^{\circ}$ and tilt $30^{\circ}$. (c) A projective transformation of $a$. a and $b$ look like the same shape with different orientations relative to the observer, but a and $c$ do not look like the same shape, although they should if shape perception was based on projective invariants.

feld (1992) showed that Equations 2 produce invariants that are more adequate for image formation. Inspection of Equations 1 shows that, in perspective, $f$ has a multiplicative effect on $x$ and $y$. This means that changing $f$ affects only the size of the image, but not its shape. On the other hand, the effect of $C$ on $x$ and $y$ is more complex, which means that changing $C$ changes not only the size but also the shape of the image. Consider an example of a square slanted around one of its sides. When the distance $C$ between the square and the observer is large relative to the size of the square (more exactly, when the distance $C$ is large relative to the range of the slanted square in depth), an image of the square is a rectangle. But if the square is brought closer to the observer, the image becomes a trapezoid. ${ }^{4}$ This means that although the retinal shape is insensitive to the distance $f$, it is sensitive to the distance $C$. Note that in the case of image formation in the eye, $f$ is constant (it is an anatomical parameter of the eye ball), whereas $C$ is variable and unknown.

Consider now Equations 2. Here $C$, but not $f$, has the multiplicative effect on $x^{\prime}$ and $y^{\prime}$. This means that, for a given retinal image, the shape on the object plane that can 
give rise to this retinal shape is sensitive to $f$, but not to $C$. These analyses imply that using inverse image formation to analyze the mapping between the object plane and image plane is more efficient for the purpose of shape analysis than is using image formation, because the shape, after applying the inverse image formation, is insensitive to distance $C$, which is variable and unknown, whereas the shape, after applying image formation, is insensitive to distance $f$, but $f$ is constant. As a result, using Equations 2 will allow formulating shape equivalence that will be insensitive to the distance $C$. This is what Pizlo and Rosenfeld (1992) did first. Next, they showed how Equations 2 can be used to derive such equivalences that are represented by perspective invariants.

\section{Perspective Invariants of Shape}

Using Equations 2, Pizlo and Rosenfeld (1992) analyzed the effect of slant $\sigma$ on the segment length and angle size for an arbitrary but constant tilt $\tau$. These effects can be approximated by quadratic functions of the form $c \cdot \sigma^{2}$, where $c$ is a constant depending on the tilt direction relative to the orientation of the segment (or angle) and depending on the length of the segment (or size of the angle). These quadratic functions were used to analyze the effect of slant on the $\Psi$ function, which is a contour-based shape descriptor. Figure 6 shows the $\Psi$ functions for the shape from Figure $5 b$ (slant0 function) and for its inverse perspective images with slant $65^{\circ}$ and tilt $30^{\circ}$ (slant65 function) and slant $75^{\circ}$ and tilt $30^{\circ}$ (slant 75 function). Note that this last function represents the shape from Figure $5 \mathrm{a}$, which simply means that Figure $5 a$ is an inverse perspec-

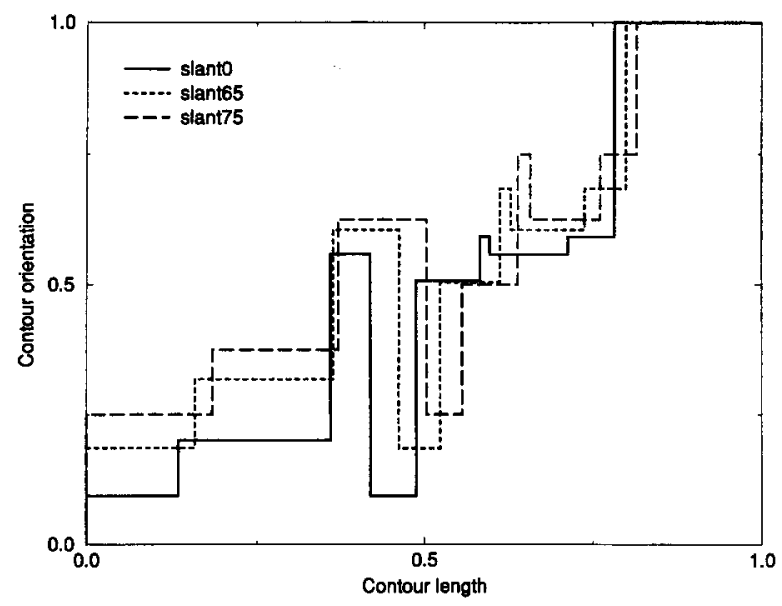

Figure 6. $\Psi$ functions. The slant0 function represents the shape in Figure 5b, the slant 75 function represents the shape in Figure $5 \mathrm{a}$, and the slant 65 function represents an inverse perspective transformation of the shape in Figure $5 b$ with slant $65^{\circ}$ and tilt $30^{\circ}$. The notation that we use here (i.e., slant0 for Figure $5 b$ and slant75 for Figure 5a) results from the method used in Pizlo and Rosenfeld's (1992) algorithm. Specifically, the algorithm works by using inverse perspective transformations, rather than perspective ones. Thus, the shape in Figure $5 \mathrm{a}$ is an inverse perspective image of the shape in Figure $5 \mathrm{~b}$ with slant $75^{\circ}$, and this is equivalent to the fact that the shape in Figure $5 \mathrm{~b}$ is a perspective image of the shape in Figure 5a with slant $75^{\circ}$. tive image of Figure $5 \mathrm{~b}$ with slant $75^{\circ}$ and tilt $30^{\circ}$. To construct a $\Psi$ function, one starts from a given point on the contour and moves along the contour, plotting the contour orientation relative to the orientation at the starting point as a function of the contour length $q$ normalized to the total contour length.

Pizlo and Rosenfeld (1992) formulated and proved the existence of two invariant properties of the $\Psi$ function, describing an arbitrary shape under inverse image formation. First, the corresponding points on the $\Psi$ functions for all slants and for a given tilt and given starting point are approximately collinear:

$$
\left(q_{k}(\sigma)-q_{k}(0)\right) /\left(\Psi_{k}(\sigma)-\Psi_{k}(0)\right)=C_{k},
$$

where $\left(q_{k}(\sigma), \Psi_{k}(\sigma)\right)$ are coordinates of a $k$ th point on the $\Psi$ function, and $C_{k}$ is a constant characteristic for a $k$ th point on the $\Psi$ function. This collinearity feature is easily seen in Figure 6 for successive steps of the functions (the steps are obviously corresponding because they represent corresponding corners of the shape). Second, for any triplet of the $\Psi$ functions, a ratio of distances between the corresponding points on the functions is approximately constant for all points along the contour. In particular, if one of these functions represents shape with $0^{\circ}$ slant,

$$
\left(\Psi_{k}\left(\sigma_{1}\right)-\Psi_{k}(0)\right) /\left(\Psi_{k}\left(\sigma_{2}\right)-\Psi_{k}(0)\right)=\sigma_{1}^{2} / \sigma_{2}^{2}
$$

Now, it will be shown how the invariant properties represented by Equations 3 and 4 can be used to recognize a shape from its single perspective image. The observer's task is as follows: assuming that the standard shape is known (more exactly, that the frontal view of the standard shape in known), the question is whether a given retinal image could have been produced by this shape with unknown slant, tilt, and distance. ${ }^{5}$ Let the slant 75 function in Figure 6 represent the standard shape (this shape is shown in Figure 5a). Let the slant0 function represent its retinal image (retinal shape) with unknown slant and known tilt (this shape is shown in Figure 5b). It is assumed that the correspondence between one point on these two shapes is known, so that their $\Psi$ functions are constructed by starting from this point. The case of unknown tilt and unknown starting point will be discussed later. Let the unknown slant be denoted by $\sigma_{1}$. Then, an inverse perspective of the retinal shape is calculated from Equations 2 for an arbitrary slant $\sigma_{2}$ and for the known tilt (here $30^{\circ}$ ). Figure 6 shows the $\Psi$ function (slant65) for the inverse perspective of the retinal shape with $\sigma_{2}=65^{\circ}$. Then, for some number of points $\left(P_{1}, \ldots, P_{n}\right)$ on the $\Psi$ function representing the retinal shape, the corresponding points on the slant65 function are determined (note that these points do not have to represent the vertices of the polygons; they can be arbitrary points along the $\Psi$ function). This is easy because the shape represented by slant 65 function has been directly computed from the retinal shape and, therefore, it is known which points on the two shapes are corresponding. Next, the corresponding points on the function for the standard shape (slant 75 function) are found using the collinearity feature (Equation 3). Then, ratios $\rho$ of distances between the corresponding points on all three func- 
tions are calculated using the Equation 4 . These ratios are approximately constant [and equal to $\left(\sigma_{1} / 65^{\circ}\right)^{2}$ ] for all $n$ points if and only if the retinal shape could have been produced by the standard shape. To verify whether this ratio is approximately constant, a histogram of $\rho$ is computed from the $n$ corresponding points, and it is checked whether its standard deviation $(S D)$ is small. Pizlo and Rosenfeld (1992) showed that the smallness of $S D$ is the necessary and sufficient condition for the retinal shape and standard shape to be equivalent in the transformation represented by image formation (Equations 1). After this equivalence is established (i.e., shape is recognized), the unknown slant can be estimated from the mean value $\rho_{m}$ of the histogram using the formula $\sigma_{1}=\left(65^{\circ}\right) \cdot \sqrt{ } \rho_{m}$. Thus, this algorithm allows not only the recognition of a shape (i.e., solving the shape constancy problem) but it also gives an estimate of its slant. Note, however, that, in this algorithm, slant representing the 3-D orientation is not used to recognize shape, as in Helmholtzian theory. Instead, slant can be estimated after shape is recognized. This makes Pizlo's (1994) theory different not only from Helmholtzian theory but also from Gibsonian theory. In Gibsonian theory, invariants can be used to recognize shape, but they do not give an estimate of slant.

If tilt is unknown, several tilts have to be tried (usually not more than four are required to recognize the shape correctly). Tilt that gives rise to the smallest $S D$ is a good estimate of the actual tilt of the object. Similarly, if the correspondence between starting points of the $\Psi$ functions for standard and retinal shapes is not known, several starting points have to be tried (usually not more than a dozen are needed).

The operation of this algorithm can be interpreted as follows: Although image formation on the retina, as described by Equations 1, is a transformation that can produce severe deformations of a shape (e.g., a square can be transformed into an irregular quadrilateral), these deformations are not completely arbitrary (e.g., a square cannot be transformed into a circle). In fact, these deformations are quite restricted: they are represented by only 3 degrees of freedom (slant, tilt, and distance). Specifically, the fact that the entire contour was subjected to the same transformation represented by Equations 1 implies that all parts of the transformed contour must share some property that represents this transformation (in Pizlo and Rosenfeld's algorithm, this common property is represented by the value of $\rho$ ). Thus, if the comparison shape is an image of the standard, the histogram of this property computed from all parts of the contour must have a small $S D$. If, however, the comparison shape is not an image of the standard, there is no transformation represented by Equations 1 that can transform all parts of the comparison shape to the corresponding parts of the standard one. In such a case, $S D$ will be large. As a result, $S D$ represents the amount of differences between the standard and comparison shapes that cannot be attributed to image formation. Such differences will be called nonperspective differences.

Note that, in this algorithm, it was important that the frontal view of the standard shape was known to the ob- server. Only then was it guaranteed that the standard and comparison shapes were equivalent under image formation, and the Equations 3 and 4 hold. If the algorithm, which was described above, was given a reference shape that was a retinal image of a standard shape with unknown slant $\sigma^{\prime}$ and tilt $\tau^{\prime}$ and then was required to recognize if another retinal image of the standard shape with unknown slant $\sigma^{\prime \prime}$ and tilt $\tau^{\prime \prime}$ (comparison shape) was produced by the standard shape, the algorithm may produce an incorrect answer because the comparison shape would be, in a general case, a nonperspective image of the reference shape and, as a result, Equations 3 and 4 will not hold. In other words, because image formation does not satisfy a closure axiom, even if the comparison shape is a retinal image of the standard and the reference shape is a retinal image of the standard, the comparison shape is not, in a general case, a retinal image of the reference shape. Instead, the comparison shape is a projective image of the reference shape. However, it was already pointed out earlier in this paper that the human visual system does not seem to involve projective invariants. It seems, therefore, that the assumption about familiarity of the frontal view of the standard shape is not too strong and that releasing this assumption may lead to perceptual effects that contradict existing psychophysical results and phenomenological observations (see Figure 5). ${ }^{6}$

It is worth pointing out, however, that, under some assumptions, the requirement that the frontal view of the standard shape be known does not have to be satisfied at all. Let the reference shape be a retinal image of the standard shape with unknown slant $\sigma^{\prime}$ and tilt $\tau^{\prime}$, and assume that when the reference shape was obtained on the retina, the range of the standard shape in depth was small relative to the distance of the shape from the observer. Under such conditions, the image formation on the retina can be approximated by an affine transformation (geometrical interpretation of affine transformation is called weak perspective, which is a composition of an orthographic transformation and size scaling). Next, assume that the observer is given a comparison shape, which is a retinal image of the standard shape with unknown slant $\sigma^{\prime \prime}$ and tilt $\tau^{\prime \prime}$ and, again, we assume that the range of the standard shape in depth was small relative to the viewing distance. Thus, the formation of this image is assumed to satisfy the affine approximation. It is known that affine transformations form a group and, hence, satisfy the closure axiom. As a result, the reference shape is an affine transformation of the comparison shape with some slant $\sigma^{\prime \prime \prime}$ and tilt $\tau^{\prime \prime \prime}$ and, therefore, the algorithm described above can be applied and the $S D$ will be small for tilt $\tau^{\prime \prime \prime}$. The only problem that can be encountered in this case is that the tilt $\tau^{\prime \prime \prime}$ for which $S D$ is small and the slant estimated by the algorithm (this estimate of slant would be approximately equal to $\sigma^{\prime \prime \prime}$ ) do not represent the actual tilt and slant of the standard shape when it produced the comparison shape on the retina. In other words, in such a case, shape will be recognized correctly, but the estimate of its 3-D orientation will be wrong. This is an important observation because it means that Pizlo and Rosenfeld's algorithm is more general than 
are the theories based on affine invariants. It can be applied to all cases where affine approximation to perspective holds (in such a case, the frontal view of the standard does not have to be known), and, furthermore, it can be applied to the cases where perspective cannot be approximated by affine (in such a case, the frontal view of the standard shape must be known). The only cases in which this algorithm cannot guarantee the correct recognition are those in which the recognition must involve establishing projective equivalence. However, it seems that human observers also cannot achieve shape constancy across projective equivalence. We can, therefore, conclude that Pizlo's theory can account better for a wider range of perceptual phenomena related to shape perception than can prior models and that the limitations of this theory seem to be psychologically plausible.

\section{Perceptual Implications of Perspective Invariants}

Pizlo (1994), in his theory of shape constancy based on perspective invariants, divided prior experiments and theories of shape perception into two complementary but separate groups. The first group is relevant to shape constancy phenomenon, which refers to the fact that the percept of the shape of a given object remains constant despite changes in the object's retinal image. The second group is relevant to what he calls "shape ambiguity" phenomenon, which refers to the problem of discriminating among shapes of objects that can give rise to the same retinal image. This distinction between shape constancy and shape ambiguity seems to have been overlooked in the past (see Thouless, 1934, for the only exception). As a result, most of the prior theories and experiments were relevant to shape ambiguity, even though the authors of these theories and experiments claimed that they were relevant to shape constancy (e.g., Beck \& Gibson, 1955; Kaiser, 1967; Leibowitz, Wilcox, \& Post, 1978; Thouless, 1931).

Because of the confusion between shape constancy and shape ambiguity, results of prior experiments seemed to be inconsistent. Most of the experiments used ellipses or triangles as stimuli, showing that accurate and precise judgments about shapes are possible only when cues, other than the shape itself, to 3-D orientation were available to the subject. These results seemingly implied that shape constancy is achieved by taking the object's orientation into account. Other experiments, however (Stavrianos, 1945), used rectangles as stimuli, showing that judgments about shape are equally accurate and precise with and without cues to 3-D orientation. These results showed that shape constancy is not based on taking the object's orientation into account. This apparent contradiction between results of prior experiments has been explained for the first time by Pizlo's (1994) theory based on Pizlo and Rosenfeld's (1992) algorithm. If shape perception is studied with stimuli that are not equivalent under perspective (e.g., rectangles), shape constancy can be achieved by applying Pizlo and Rosenfeld's algorithm, and the object's orientation relative to the observer does not need to be taken into account (this is similar to Stavrianos's, 1945, result). If, however, shape perception is studied with shapes that are equivalent under perspective transformation in general, or under image formation in particular (e.g., ellipses or triangles), the shape ambiguity problem is produced, and Pizlo and Rosenfeld's algorithm cannot discriminate among the shapes because each shape could produce a given retinal image. But, if the 3-D orientation of the stimulus (i.e., slant and tilt) can be reconstructed from cues other than shape, the algorithm can discriminate among the stimuli by comparing the slant and tilt estimated from shape with the slant and tilt estimated from the other, independent cues. In other words, for a given retinal image, the algorithm will produce a set of possible recognitions in the following form: stimulus 1 with slant $\sigma_{1}$ and tilt $\tau_{1}$, stimulus 2 with slant $\sigma_{2}$ and tilt $\tau_{2}, \ldots$, stimulus $n$ with slant $\sigma_{n}$ and tilt $\tau_{n}$. Now, let the orientation $\left(\sigma_{k}, \tau_{k}\right)$, estimated from shape by Pizlo and Rosenfeld's algorithm, agree with the orientation recovered from other, independent cues. This fact means that the given retinal image was produced by stimulus $k$ and this is the correct response (note that this method will uniquely determine the shape; i.e., Euclidean structure up to size scaling). Thus, if cues to 3-D orientation, other than the shape itself, are present, Pizlo and Rosenfeld's algorithm can discriminate among the stimuli (this is similar to Thouless's, 1931, results).

Consider now limitations of Pizlo's perspective invariants theory in natural vision. The perspective invariants can be applied to the image of a planar, or approximately planar, contour (or contours). Note that the contour does not have to be a polygon; it can also be a smoothly curved shape. In the case of a polygon, the polygon should have four or more vertices. However, triangles, or objects with triangular faces, can also be recognized if tilt of the shape is known, at least approximately (see Pizlo \& Rosenfeld, 1992 , for an example). If the algorithm is applied to polyhedra with several faces, the recognition of the individual faces can often be sufficient to recognize the entire object. Sometimes, however, the information about how the individual faces are arranged with respect to one another is needed (Pizlo \& Rosenfeld, 1992).

Next, consider implications of the requirement that the object to be recognized has planar, or approximately planar, contours. If this requirement is not satisfied, Equations 3 and 4 do not hold and recognition may be inaccurate. This certainly restricts the family of objects that can be recognized. Note, however, that this restriction does not seem to be too strong. The objects that cannot be recognized according to this theory are only those that have no flat or approximately flat contours. In fact, existing results on shape recognition by human observers in the case of 3-D wire or star objects provide support for this restriction (Edelman \& Bulthoff, 1992; Rock \& DiVita, 1987; Rock et al., 1989).

Consider now some predictions produced by perspective invariants theory of shape constancy. The invariants are insensitive to slant, but they are sensitive to tilt: if tilt is not known, it has to be searched for. This, however, requires some additional time. If such time is not allowed, the response is likely to be incorrect. These effects of slant and tilt were tested in two experiments reported by Pizlo 
(1994). In Pizlo's experiments, the subject was required to decide whether a comparison quadrilateral was a perspective image of the standard quadrilateral (all quadrilaterals were convex). The size of the stimulus was about $1 \mathrm{~cm}$, and the viewing distance was $50 \mathrm{~cm}$. As a result, the simulated range in depth of the comparison stimulus was not greater than 0.02 of the viewing distance and, thus, the perspective transformation of the standard shape could be almost exactly approximated by an affine transformation. The tilt and slant randomly varied from trial to trial, and both the ranges of possible slant and tilt values and their limits were known to the subject. Two ranges of slant and tilt values were used: one range was narrow and the other range was wide (see Pizlo, 1994, for details of the method he used). According to Pizlo's theory, the random variability of slant does not affect performance because the knowledge of slant is not needed to recognize shape. The random variability of tilt, however, may affect performance because, in the theory, the tilt must be known or it has to be searched for. Thus, if the range of possible tilt values was narrow, any tilt value from within this range could lead to a correct recognition. However, if the range of possible tilt values was wide, a random tilt chosen from within this range was more likely to lead to an incorrect response. According to the theory in which shape constancy is based on projective invariants, the subject's performance should be at chance level in all conditions because all convex quadrilaterals are projectively equivalent. According to the theory based on affine invariants, on the other hand, the subject's performance should be close to perfect and the same in all conditions simply because affine invariants could be used in the case of quadrilaterals and the invariants do not involve the information about slant and tilt.

The subject's performance was measured by a speedaccuracy tradeoff function (SATF), and the effect of experimental conditions on this function was analyzed. Pizlo and Rosenfeld's algorithm was tested on the same stimuli, and the experimental and simulation performance were compared. Results of these experiments showed that the subject's performance is not affected by the width of the possible slant values. However, the performance was affected by the width of possible tilt values: wider range lead to worse performance (i.e., shallower SATFs, which represent longer reaction times and higher error rates). All these results agree with predictions of Pizlo's theory, and they contradict prior theories based on projective or affine invariants or based on cues to 3-D orientation. Furthermore, it was shown that Pizlo and Rosenfeld's (1992) algorithm, which is a computational model of shape constancy in Pizlo's theory, gives rise to exactly the same pattern of results as observed in psychophysical experiments.

Despite the fact that Pizlo's theory is consistent with a wide range of existing psychophysical results, its psychological plausibility should be further tested. Specifically, it must be verified whether the results from shape constancy experiments with simple 2-D shapes can generalize to more complex 2-D shapes and to 3-D shapes. The example shown in Figure 3 suggests that this is the case. However, this observation should be tested in psychophysical experiments. Such experiments will be described next.

In the first experiment, we tested shape constancy for complex 2-D shapes (random polygons) and, further, we measured the psychometric function for the discrimination between a perspective and nonperspective image of a given standard shape. In the second experiment, we tested whether adding a textured background can improve the discrimination performance. The third experiment generalized the results from the second experiment to 3-D shapes (cuboids, cylinders, tetrahedrons, and slanted pictures of cuboids). Finally, in the fourth experiment, we tested planar motion as a cue to 3-D orientation in distance discrimination task. Testing planar motion was important because adding motion to some stimuli in Experiment 3 resulted in perceived nonrigidity. Such nonrigidity implied that motion is not a reliable cue to 3-D orientation.

\section{EXPERIMENT 1 \\ Discrimination Between a Perspective and Nonperspective Image of a Random Polygon}

Experiment 1 tested two aspects of Pizlo's (1994) theory. First, it tested human shape constancy by using complex 2-D shapes-namely, random polygons. Second, it tested whether the theory provides a perceptual dimension that underlies shape constancy. Specifically, if $S D$, the measure of nonperspective differences in Pizlo and Rosenfeld's algorithm (1992), underlies shape constancy, $S D$ should represent a perceptual dimension that is used to discriminate between a perspective and nonperspective image. If this is the case, the relation between the proportion of responses "nonperspective" and $S D$ (or some function of $S D$ ) would be a psychometric function having the shape of a cumulative Gaussian distribution. This experiment was designed to measure such a psychometric function. To our knowledge, this is the first attempt to measure a psychometric function for shape perception in general and shape constancy in particular. In the past, such attempts have been made only for a very limited class of shapes, such as triangles, and the shapes were always in the frontal plane (e.g., Pizlo, 1988).

\section{Method}

Subjects. Three subjects (the 2 authors and 1 other) were tested. The third subject (J.M.) was naive with respect to the hypotheses being tested. Z.P. and M.S.G. knew all details of the experimental method. J.M. knew only some details of the method (see below). Z.P. and J.M. had prior experience as subjects in psychophysical experiments. Z.P. and J.M. were myopes, and they used their normal correcting glasses. M.S.G. was an emmetrope. Z.P. and M.S.G. received extensive practice before being tested.

Stimuli. The stimuli were line drawings of random polygons shown on a monitor $(1,152 \times 900)$ of a SUN computer (SPARC 2GS). The luminance of the contour was $56.7 \mathrm{~cd} / \mathrm{m}^{2}$, and the luminance of the background was less than $0.01 \mathrm{~cd} / \mathrm{m}^{2}$. The stimuli were viewed in a dark room. The polygons were generated using a modified version of Larsen's (1985) algorithm. Specifically, for a given center, a 


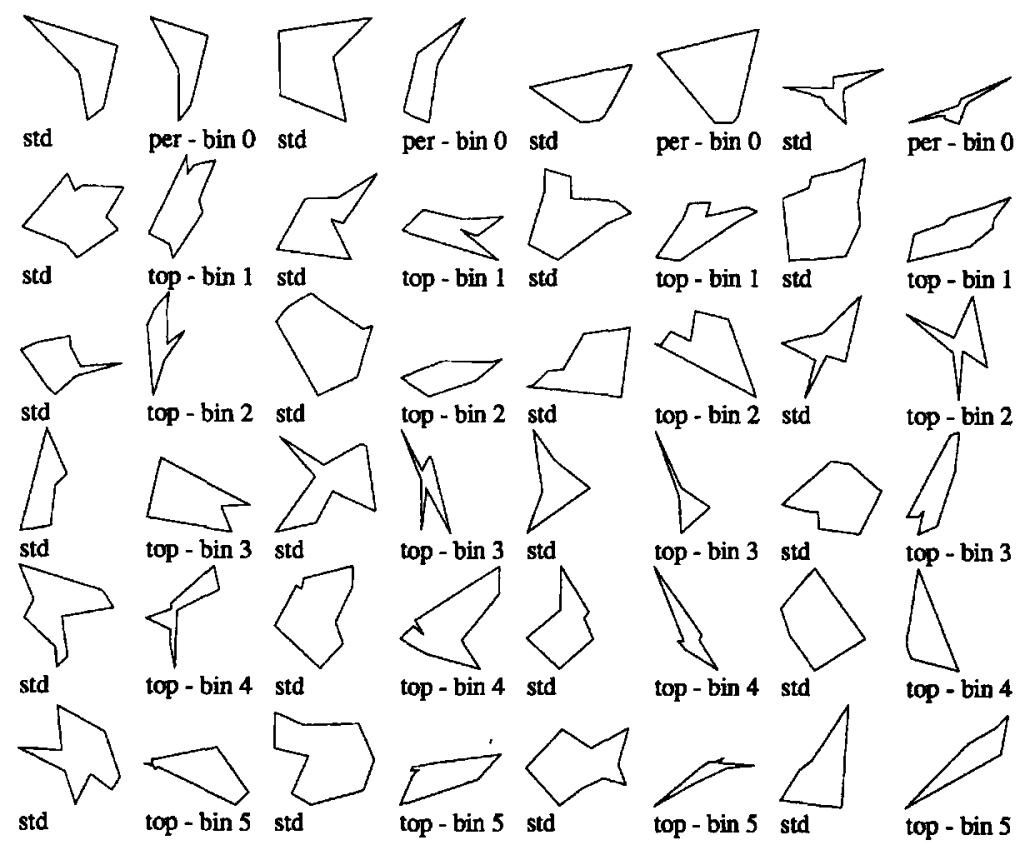

Figure 7. Examples of stimuli used in Experiment 1. The six rows show standard shapes (std) and the corresponding comparison shapes for each of the six sets of shapes. In the first row, the comparison shapes are perspective transformations of the standard (per-bin0). In the other five rows, the comparison shapes are nonperspective (topological) transformations of the standard representing all five levels (bins) of nonperspective differences. The higher the bin number, the larger the nonperspective differences between the standard and comparison shapes, as measured by Pizlo and Rosenfeld's algorithm.

set of $k$ angles (in the range from $0^{\circ}$ to $360^{\circ}$ ) and $k$ radii were randomly selected ( $k=5,7$, or 9). Each pair (angle and radius) represented one vertex of a polygon in the polar coordinate system. The set of $k$ such pairs specified $k$ vertices of a polygon. The number of vertices $(5,7$, or 9 ) varied randomly from trial to trial (Figure 7 shows examples of such polygons). The standard and comparison polygons were shown simultaneously. The standard polygon was shown in the top of the screen. The comparison stimulus, which was shown in the bottom of the screen, was either a perspective or nonperspective transformation of the standard. The position and size of the standard polygon were random. Without such randomization, the comparison polygon would be always in the similar position and with the similar size as the standard for a perspective transformation, but not for a nonperspective transformation. As a result, the position and size of the comparison stimulus would serve as artifactual cues.

The perspective stimulus was obtained by applying a perspective transformation to the standard with random tilt (in the range from $0^{\circ}$ to $360^{\circ}$ ) and random slant (in the range from $50^{\circ}$ to $80^{\circ}$ ). The distance used in the calculation was equal to the actual distance of the subject from the screen $(1.5 \mathrm{~m})$. As a result, the retinal image produced by the perspective stimulus on the screen was equivalent to the image that would be produced on the retina if the standard shape was actually slanted in 3-D (see Figure 7, for examples of perspective stimuli).

The nonperspective stimulus was computed as a perspective image of a polygon, which was a topological transformation of the standard. The topological transformation was specified as follows. Recall that the vertices are coded in the polar coordinate system as a set of pairs: radius and the corresponding angle. Assume that the vertices are numbered from 1 to $n$ ( $n$ is the number of vertices in the polygon) according to the magnitude of angles in a clockwise direction. Next, two vertices $i$ and $j(j>i+1)$ of the standard polygon were randomly chosen. Then, radii for vertices with numbers $(i+1$, $\ldots, j-1)$ or with numbers $(j+1, \ldots, n$ and $1, \ldots, i-1)$ were mul- tiplied by a constant factor chosen randomly from the range of 1 to 11. Note that this transformation preserved the number of vertices of the polygon. After performing this transformation, it was verified that convex angles were transformed into convex ones and concave angles into concave ones. If this constraint was violated another transformation was made. Furthermore, if at least one segment of the polygon was too short to be noticed (less than 10 pixels [about $7 \mathrm{~min}$ of arc]), another transformation was made. Finally, the angles were not allowed to be too close to $180^{\circ}$, since, in these cases, the presence of the vertex could go unnoticed. Angles in the range $170^{\circ}-190^{\circ}$ were considered to be too close to $180^{\circ}$ (see Figure 7, for examples of nonperspective stimuli). All these constraints were used to remove simple local features that would make the discrimination task trivial. For example, if the standard polygon was convex (all angles less than $180^{\circ}$ ), but the nonperspective polygon was concave (at least one angle greater than $180^{\circ}$ ), the correct response could be made by checking the convexity of this angle. However, checking the convexity of an angle requires analyzing local parts of the polygon (angles) without analyzing their global relationship. In such a case, a correct response would not be an indication of achieving shape constancy.

The size of the standard and comparison shape was about $10 \mathrm{~cm}$. Thus, the ratio of the simulated range in depth of the comparison stimulus to the $1.5-\mathrm{m}$ viewing distance was about 0.06 . This means that although the perspective transformation of the comparison shape was not equivalent to an affine transformation, it was nevertheless quite close to an affine transformation. ${ }^{7}$

There were six sets of trials in each session, 100 trials per set. In the first set, perspective shapes were used as comparison stimuli. In the remaining 500 trials, nonperspective shapes were used as comparison stimuli. The stimuli from all six sets were presented in a random order. The sets of nonperspective shapes were characterized by the amount of nonperspective differences between the standard and the comparison stimulus, as measured by $\log (m d e v)[\log (m d e v)=$ 
$-1.55,-0.95,-0.35,0.25,0.85) .{ }^{8}$ More exactly, each set of nonperspective shapes was represented by a range of $\log (\mathrm{mdev})$, rather than by a single value of $\log (m d e v)$ (the width of each range was equal to $0.3)$. As a result, the first set of shapes had $\log (m d e v)$ from the range $(-1.7 ;-1.4)$, the second set of shapes had $\log (m d e v)$ from the range $(-1.1 ;-0.8)$, and so on. Using a range of mdevs was necessary because there is no analytical formula that would allow distorting a standard shape to obtain a given value of nonperspective differences, as measured by mdev. Therefore, transformations with random values of parameters were applied to standard shapes and then $m d e v$ was calculated, checking to which range, if any, this comparison shape falls.

The calculation of mdev involved searching for the tilt of the comparison shape. This was done by checking 36 tilts in $10^{\circ}$ steps and choosing the tilt that produced the smallest $m d e v$. The search for the tilt value was necessary because perspective invariants are insensitive to slant, size, and distance, but not to tilt (see Perspective Invariants of Shape section above). This calculation was repeated until 100 shapes were obtained in all five sets. (Generation of the stimuli on the SPARC 2GS for a single session took about $30 \mathrm{~min}$.) Figure 7 shows examples of nonperspective stimuli from all five sets corresponding to different values of $\log (m d e v)$.

The set of perspective shapes was characterized by the same range of $\log (m d e v)$ as the first set of nonperspective shapes-namely, $(-1.7 ;-1.4)$. This means that the first set of nonperspective shapes contained shapes that were equivalent to perspective shapes, according to Pizlo and Rosenfeld's (1992) invariants. A question is, however, whether these nonperspective shapes are also perceptually equivalent to perspective ones.

Procedure. The method of constant stimuli was used. The subject's task was to respond whether or not the comparison shape was a perspective image of the standard shape. Before the actual experiment, each subject received some practice to become familiar with the stimuli and the task. The experimental session was preceded by a practice session with 60 trials ( 10 trials per each of the six sets of comparison stimuli). In each session, the subject was asked to adopt a response criterion, so that the proportion of correct responses in perspective trials was close to $100 \%$. After the block of practice trials, the subject was informed about the proportion of correct responses on trials where the comparison was a perspective transformation of the standard, so that the subject had an opportunity to adjust the response criterion as required. After each practice and experimental trial, feedback about the accuracy of the response was given. The duration of the trial was unlimited: the stimuli disappeared after the subject responded. The inspection time was usually less than $5 \mathrm{sec}$. Each subject was tested in two sessions. One session lasted about $1 \mathrm{~h}$.

The subject viewed the stimuli with one (right) eye. The head was supported by chin-forehead rest. The orientation of the screen was adjusted so that the subject's line of sight was perpendicular to the screen. The subject responded with the right hand, using a computer mouse.

The naive subject, J.M., knew that there were six sets of shapes, 100 shapes in each set. He was told that comparison shapes in 100 trials were exactly perspective images of the standard and that comparison shapes in each of the five remaining sets of trials were not exactly perspective images. He knew that some comparison shapes would appear very different from the corresponding standard shapes, whereas other comparison shapes could be quite similar to the standard shapes.

\section{Results and Discussion}

Figure 8 shows results from all 3 subjects. The abscissa shows $\log (m d e v)$, which is a measure of the amount of the
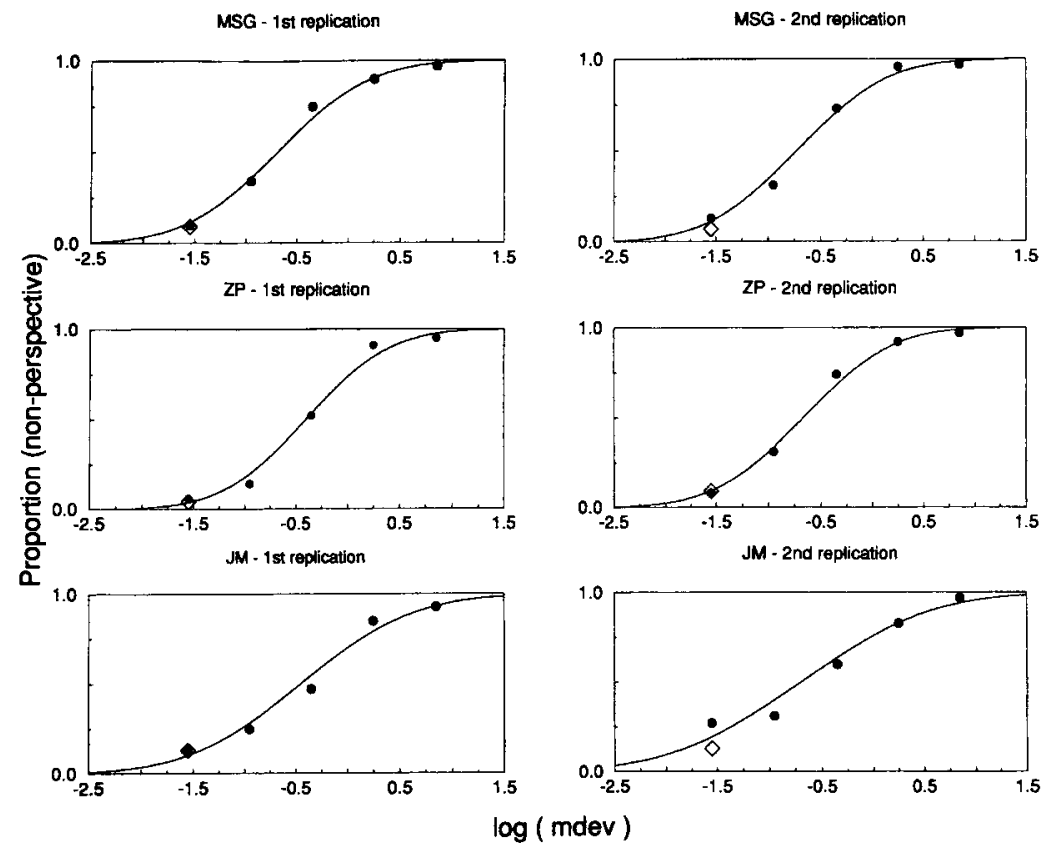

Figure 8. The psychometric functions for all 3 subjects in Experiment 1. The abscissa shows $\log (m d e v)$, which is a measure of nonperspective differences between the standard and comparison shapes in Pizlo's theory. The ordinate shows the proportion of responses "nonperspective." Circles represent results from the trials where the comparison shape was a nonperspective transformation of a standard, and the diamond represents results from trials where the comparison shape was a perspective transformation of the standard. The continuous line is the best-fitting cumulative Gaussian distribution function. 
nonperspective differences between the standard and comparison shapes. The ordinate shows the proportion of responses "nonperspective." Circles represent nonperspective trials, and the diamond represents the perspective trials.

It can be seen that the subjects could discriminate between a perspective and nonperspective image of a standard polygon. More specifically, when $\log (m d e v)$ increases, the proportion of responses "nonperspective" monotonically increases. This relationship can be represented by a cumulative Gaussian distribution function. The parameters of this function were calculated using probit analysis. In this analysis, only the data points from nonperspective trials were used. The fit was rather good, as measured by a chisquare test: in two sessions, $p>.1$ (in one session for each author). In the remaining four sessions, $p>.025$. This means that there were no large heterogeneities in the departures of the data points from the approximating curve, ${ }^{9}$ which can be seen in Figure 8 where the data points are quite close to the approximating curves. The small variability of the data points around the approximating curves means that the probability of the subjects' response "nonperspective" can be completely predicted by the value of $\log (m d e v)$.

Next, note that the point representing perspective trials coincides with, or is very close to the point representing the first set of nonperspective trials. According to perspective invariants, in both these sets of trials, the amount of nonperspective differences between standard and comparison shapes, as measured by $\log (m d e v)$, was the same and small. The fact that the two experimental points almost coincide suggests that these two sets of trials were equivalent not only geometrically but also perceptually. However, this experimental evidence is only indirect. To directly verify the claim that the two kinds of shapes, perspective and nonperspective, with the same small value of $\log (m d e v)$ are perceptually equivalent, a discrimination experiment should be used where only these two groups of shapes would be presented.

All these results show that the perspective invariants that were used in Pizlo's (1994) theory of shape constancy can account quite well for the subject's performance in the task of the discrimination between a perspective and a nonperspective image of a given shape. More specifically, $\log (m d e v)$, which is the measure of nonperspective differences in the theory, was sufficient for predicting the subject's performance in this task. The good fit of the approximating curve to the data points means that all (or almost all) variability in the subject's responses can be accounted for by variability of only one predicting variable - that is, $\log (m d e v)$ - and no other independent variable is needed. This suggests that $\log (m d e v)$ represents a perceptual dimension that is used by a human observer to solve the shape constancy problem.

Consider now one interesting implication of these results. The shape of a random polygon is a multidimensional property. Specifically, in our experiments, each shape was represented by up to 18 parameters $(x$ and $y$ coordinates of nine vertices). However, shape constancy, which involves discriminating between two such multidimensional properties, seems to be solved in only one di- mensional space defined by $\log (m d e v)$. Thus, Pizlo's (1994) theory is different from traditional theories based on the concept of metric spaces, in which dissimilarity between two objects is measured as a distance in the space used to represent these objects. In the case of shape constancy, such traditional theories cannot be used, because it is not clear whether the space for representing an arbitrary shape exists, and, if it does, how many dimensions this space should have. It is also not clear whether the number of dimensions is finite. In Pizlo's theory, this is not a problem, because the theory uses a 1-D space for shape differences, rather than a multidimensional space for shapes themselves. We want to point out that the unidimensionality of the hypothesized perceptual mechanism underlying shape constancy is not likely to be a simple artifact of the unidimensionality of the responses (perspective vs. nonperspective). The task used in this experiment belongs to the class of unordered discrimination tasks (Luce \& Galanter, 1963), where the subject is asked to decide whether the comparison and standard stimuli match with respect to one or more attributes and the subject is allowed to use only two responses: either "yes" or "no." The subject can produce such unidimensional responses regardless of how many dimensions are involved in the differences between the stimuli.

Consider now alternative explanations of our results. Helmholtzian theory cannot explain our results because the comparison stimulus was represented by contour only and, hence, it did not provide adequate cues to 3-D orientation. We want to point out, however, that a perspective image of a polygon provides some cues to 3-D orientation. Namely, the image is compressed along the direction of tilt and the amount of compression is determined by slant (Stevens, 1983; Witkin, 1981). But estimation of slant and tilt from these kinds of cue involves very strong assumptions (e.g., that all 2-D orientations in the contour of the standard shape are equally probable, as in the case of a circle). However, we used random shapes, and, therefore, this assumption was usually not satisfied. One possible argument that can be used to revive Helmholtzian theory is that although the image of a random polygon does not allow exact estimation of the 3-D orientation, perhaps this estimation is good enough to perform the shape discrimination in our task. This argument was tested (and will be shown to be wrong) in Experiment 2.

Another alternative explanation involves affine or projective invariants (Gibsonian theory). In fact these invariants could be used to discriminate whether one shape is or is not a perspective image of another, in our experiment because: (1) the perspective shape was in fact an affine (and projective) transformation of the standard, and (2) the nonperspective shape was a non-affine (and nonprojective) transformation of the standard. However, affine or projective invariants do not provide any measure of nonperspective differences. Thus, there is no theory at this moment, based on conventional invariants, that could account for the relations shown in Figure 8.

Finally, the observer could solve the task by using some version of an alignment method (Huttenlocher \& Ullman, 
1988; Lowe, 1987). Specifically, one could take an arbitrary triple of noncollinear points in the standard shape and the corresponding triple in the comparison shape and from this information determine uniquely the 3-D orientation (note that whereas three noncollinear points uniquely determine the 3-D orientation in the case of affine transformation, four points are needed in the case of perspective transformation; see Wolfe, Mathis, Sklair, \& Magee, 1991). Then, one could compute an image of the standard shape with the estimated orientation and check whether this image is identical (aligned) with the comparison shape. Again, although this method could lead to accurate discrimination in our experiment, it is not clear how to measure differences between the two images so that the amount of nonperspective differences can be estimated.

We want to point out that although the existing versions of the theories based on conventional invariants or on alignment cannot account for results of this experiment (because the theories do not provide any measure of nonperspective differences), the results do not directly contradict these theories. For example, it is possible to add some measure of differences between two shapes (e.g., the amount of non-overlap, as used by Hong \& Tan, 1989) to the theories based on conventional invariants or alignment so that the theories could account for the fact that the more different the shapes are, the more likely is the response nonperspective. However, it is not clear whether the theories after this elaboration could produce monotonic relationships, such as the ones shown in Figure 8, and whether these relationships could be approximated by a cumulative Gaussian distribution function, which is a conventional psychometric function. Psychological plausibility of these theories will be tested directly in Experiment 3 by verifying predictions of the existing versions of these theories. It will be shown that despite the fact that the theories, after some elaborations, could perhaps account for results of the present experiment, they are not adequate models of human 3-D shape perception.

Having established that shape constancy can be achieved even though the shapes are represented by contour only, it remains to be tested whether conventional cues to 3-D orientation can contribute to shape constancy. It was shown by Thouless (1931) that cues to 3-D orientation were beneficial in judgments about shapes of ellipses. However, as pointed out earlier in this paper, Thouless's experiments tested a very special case, called by Pizlo (1994) "shape ambiguity" because all his stimuli (ellipses) were perspectively equivalent, which means that the retinal image of a stimulus did not allow discrimination among the stimuli. Therefore, the results from Thouless's shape ambiguity experiment may not generalize to other stimuli. In fact, Stavrianos (1945), who used rectangles that are not perspectively equivalent, found that cues to 3-D orientation were not beneficial in judgments about shape. Experiment 2 was designed to test whether the results of Stavrianos generalize to the case of random polygons with more than four sides.

\section{EXPERIMENT 2 The Role of Texture in Shape Discrimination}

\section{Method}

Subjects. Three subjects (the 2 authors and 1 other) were tested. The third subject (B.M.) was naive with respect to the hypotheses being tested and the method of designing the stimuli. B.M. was a myope and used his normal glasses in the experiment. M.S.G. and Z.P. were tested in two replications of each condition, and B.M. was tested in one replication.

Stimuli. In this experiment, the stimuli were designed in the same way as those in Experiment 1 except for the following modifications. In the second and third condition, a perspective image of a textured surface (square grid) was superimposed on the image of the comparison shape, so that the comparison shape looked as if it was lying on a flat surface represented by this texture. The image of the grid served as a cue to 3-D orientation. It has been claimed in the past that the image of a texture in general (Gibson, 1979; Witkin, 1981) and of a rectangle or square in particular (Haralick, 1989; Stevens, 1983) is very useful in reconstructing slant and tilt of a plane. Therefore, it seemed reasonable that testing the role of a square grid as a cue to 3-D orientation would shed more light on the general problem of taking the 3-D orientation of an object into account in shape perception. Using square grid as a background also allows the testing of some specific predictions of Helmholtzian theory described in the previous section. In the previous section, it was pointed out that the contour of the comparison stimulus might have been used to estimate (approximately) the 3-D orientation, which then could be used to reconstruct the shape. This reconstruction could not be accurate, however, because random shapes that were used in Experiment 1 did not allow accurate estimation of the 3-D orientation. Using square grid as a cue to 3-D orientation further tests Helmholtzian theory because this grid does allow accurate estimation of the 3-D orientation. Therefore, if subjects use 2-D orientation of lines in the image as a cue to 3-D orientation (as in all theories cited in this paragraph), the percept should benefit from the presence of texture.

Procedure. The signal detection method was used. In all three conditions, the perspective shapes were generated in the same way as in Experiment 1. The nonperspective shapes were used only in the first two conditions. These shapes were generated so that $-0.7<$ $\log ($ mdev $)<-0.4$. From the results of Experiment 1, it can be predicted that when only contour of the shape is present, the discrimination between a perspective and a nonperspective image should lead to $d^{\prime}$ approximately equal to 1.5 for Z.P. and M.S.G. (these 2 subjects served in both experiments). Each session consisted of 600 trials ( 300 perspective and 300 nonperspective shapes). The subject's task was to decide whether the comparison shape was a perspective image of the standard. The first condition was very similar to that of Experiment 1: discrimination between perspective and nonperspective shapes represented by contour. In the second condition, a perspective image of a square grid was shown along with the comparison shape. The simulated 3-D orientation of the grid and of the comparison shape were identical. To avoid 2-D local cues, such as the relative orientation of the contour parts relative to the grid, the 2-D orientation of the grid was randomized before the perspective transformation was applied.

In the third condition, all comparison shapes were perspective shapes. However, the square grid background was "rotated" in 3-D either with the same tilt as the comparison shape (same rotations) or with different tilt (different rotations). Slant of the grid was always the same as slant of the comparison shape. As in the second condition, the 2-D orientation of the grid was randomized before the perspective transformation was applied. There were 300 trials with the same rotations and 300 trials with different rotations. The subject's 
task was to decide whether the tilt of the grid was the same as the tilt of the comparison shape.

Comparing the performance in the first and second conditions reveals whether the subject used texture as a cue to $3-\mathrm{D}$ orientation in shape perception, and comparing the first and third conditions reveals whether the subject had the perceptual capacity to use texture as a cue to the 3-D orientation in shape perception. If the subject can reliably use the 3-D orientation derived from texture to reconstruct the shape, the subject will be able to decide whether the tilt provided by texture is correct or not, by comparing the reconstructed shape with the standard shape. The third condition was needed because, in the second condition, the subject might choose not to use the texture even if the subject had the perceptual capacity to do so. As a result, the performance in the second condition might not represent the subject's perceptual capacity, but only perceptual strategy. The third condition tests directly this perceptual capacity to use the 3-D orientation in shape perception. Note that the third condition does not test whether the subject can reliably perceive the 3-D orientation from texture, but whether the 3-D orientation derived from texture can be used in shape perception (perception of the 3-D orientation from texture could be tested in a task where the 3-D orientation of one textured plane would be discriminated from the 3-D orientation of another textured plane).

The parameter that remains to be specified in the third condition is the difference between the tilt of the background and the tilt of the comparison shape. If this difference is too small, performance would be at chance level. If this difference is too large, performance could be perfect. To be able to make direct comparison among the three conditions, one should use such difference $\Delta \tau$ between the tilts in the third condition so that, under the assumption that the subject can reliably integrate the 3-D orientation with shape, this condition would be as difficult as the first condition. Note that Pizlo and Rosenfeld's (1992) algorithm provides a computational tool, which can be used to determine $\Delta \tau$. Namely, if the tilt of the background is the same as the tilt of the comparison shape, the tilt estimated from the background can be used to recognize the comparison shape; in such a case, mdev will be close to zero. If, however, the tilts of the grid and of the comparison shape are different, $m d e v$ will not be close to zero. Let $\log (m d e v)$, in the latter case, be in the range from -0.7 to -0.4 , which is exactly the same range as that used in generating the nonperspective shapes in the first two conditions. The values of $\log (m d e v)$ from this range are obtained when $\Delta \tau$ is, on the average, equal to $24^{\circ}$. In such a case, if the subject could derive the tilt from the background in the third condition, the discrimination performance, as measured by $d^{\prime}$, should be the same as that observed in the first condition.

The exposure duration of the stimulus was constant and equal to $5 \mathrm{sec}$. Constant duration was used because the naive subject (B.M.) tended to deliberate very long in the third condition, which increased the duration of the sessions substantially. Very long sessions were not desirable because the results could be affected by fatigue of the subject. Also, large differences in viewing time across conditions could introduce a confounding factor related to the speed-accuracy tradeoff (e.g., Luce, 1986). For example, if $d^{\prime}$ in the third condition was higher than $d^{\prime}$ in the first condition, this could be produced by the subject's tendency to respond quickly in the first condition, rather than by the fact that the third condition was easier. We want to point out, however, that limiting viewing time to $5 \mathrm{sec}$ did not produce any noticeable deterioration in results, including results in the third condition for Subject B.M. After the set of practice trials, the subject was informed about the proportion of correct responses on both groups of trials (perspective vs. nonperspective shape in Conditions 1 and 2, and same vs, different 3-D orientations in Condition 3).

All other features of the experimental method were the same as those in Experiment 1.

\section{Results and Discussion}

The results from all three subjects are shown in Figure 9. First, note that $d^{\prime}$ in the first condition for Z.P. and
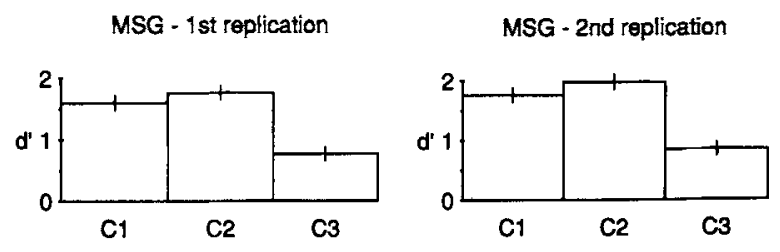

ZP - 1st replication

ZP - 2nd replication
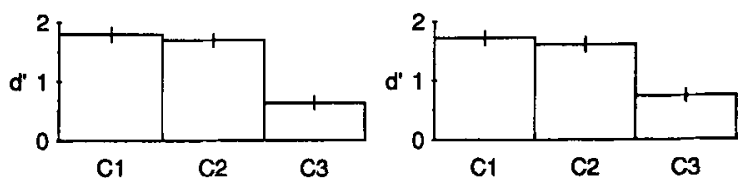

BM

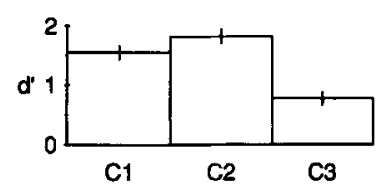

Figure 9. The estimates of $d^{\prime}$ for all 3 subjects in the Experiment 2 (the standard errors of $d^{\prime}$ are indicated by the vertical line segments). C1: discrimination between perspective and nonperspective shapes represented by contours. $\mathrm{C} 2$ : same as $\mathrm{C} 1$, but with a square grid as a cue to the $3-D$ orientation of the comparison shape. $\mathrm{C} 3$ : discrimination between the 3-D orientation of a comparison shape and that of a square grid.

M.S.G. is about 1.7 (standard error about 0.12 ), which is very close to the value predicted for these subjects from the results of Experiment 1 where the stimuli were the same and the method of constant stimuli was used (performance of the third, naive subject, was similar). This means that the results of the discrimination did not depend on which psychophysical method was used. Second, note that $d^{\prime}$ in the second condition is very close to $d^{\prime}$ in the first condition; the differences are comparable to the standard errors of the individual estimates. Moreover, the differences are not even consistent: for 2 subjects, adding the textured background improved performance slightly, but, for the third subject, performance deteriorated slightly.

These results are consistent with our interpretation of the results of Experiment 1. Namely, perception of shape is not based on taking the 3-D orientation into account, but it is most likely based on invariants. Furthermore, note that these results contradict the alternative explanation based on the assumption that perception of shape is based on 3-D orientation estimated from the amount of compression of the contour of the comparison shape, as suggested by various theories and methods (e.g., Stevens, 1983; Witkin, 1981). As pointed out in the discussion of Experiment 1, this estimate of the 3-D orientation is not reliable when random shapes are used. Therefore, if perspective image of some regular contours (such as a square grid) are shown, the subject could estimate the 3-D orientation much more reliably and, hence, perform much better in shape discrimination. The fact that in the second condition of Experiment 2, where a square grid was added as a background, performance did not improve, relative to 
that in the first condition, suggests that compression of the contour in the image of the comparison shape is not used as a cue in shape perception.

Consider now performance in the third condition. In this condition, $d^{\prime}$ was about 0.75 . Recall, that the mean difference in tilt between the square grid and the comparison shape was $24^{\circ}$. Thus, the threshold estimated for $d^{\prime}=$ 1 in this task was about $32^{\circ}$, which means that the subjects could not reliably discriminate the tilt of the background from that of the comparison shape. Note that the threshold $\left(32^{\circ}\right)$ in our tilt discrimination task is larger than the threshold estimated by Stevens (1983). (However, it is not possible to make direct comparison with Stevens's results because he calculated thresholds and systematic errors separately from each stimulus and condition, rather than from all of them. The systematic error of tilt judgments in Stevens's experiment was up to $6^{\circ}$ and threshold was up to $12^{\circ}$. Note that computing threshold from all stimuli and conditions together is likely to increase the value of the estimated threshold.) The difference between thresholds measured by Stevens and thresholds measured in our experiment is likely to be related to the fact that Stevens tested perception of 3-D orientation, whereas we tested perceptual integration of 3-D orientation and shape, which is an essential element in Helmholtzian theory of shape constancy. Thus, the poor discrimination performance that we observed implies that the subjects could not reliably integrate the information from texture and from contour, which contradicts Helmholtzian theory. It has to be pointed out, however, that before Helmholtzian theory is rejected, results from texture have to be generalized to other cues to 3-D orientation. Experiments 3 and 4 will provide further evidence generalizing the results of Experiment 2 and further contradicting Helmholtzian theory.

Compare now the performance in the third condition with that in the first two conditions. It can be seen that $d^{\prime}$ in the third condition was much lower than $d^{\prime}$ in the first two conditions. This difference is large, relative to the standard errors. Recall, that the stimuli in the third condition were designed in such a way that if the subjects could effectively use tilt from texture, the performance in this third condition should be identical to that in the first condition. But this was not the case. As a result, the difference between the performance in the third condition and the first two conditions represents the degree to which the mechanism based on taking the 3-D orientation into account is less reliable than the mechanism based on perspective invariants. We want to point out that this interpretation is based on the assumption that Pizlo and Rosenfeld's algorithm is an adequate model of shape perception, because the difference between tilts in the third condition was chosen by using this algorithm. Despite the fact that Pizlo and Rosenfeld's algorithm is consistent with a wide range of experimental results, this particular interpretation should be further tested using other stimuli. Such a test was performed in Experiment 3 for 3-D shapes.

To summarize, results of Experiment 2 show that the subjects' performance in the discrimination between a perspective and a nonperspective image of a standard shape does not benefit from the presence of a cue to 3-D orientation (texture) because the subjects cannot effectively use this cue in shape perception. This is consistent with prior results where it was shown that adding cues, which provided information about the slant, did not improve performance in shape matching (Stavrianos, 1945) and that the human observer cannot reliably reconstruct 3-D Euclidean or similarity structure of an object from cues to 3-D orientation and position (Todd \& Bressan, 1990; Todd \& Norman, 1991, 1994; Todd \& Reichel, 1989). Thus, our results from the first two experiments, along with prior results, contradict Helmholtzian theory and support Pizlo's theory based on perspective invariants.

In Experiment 3, the results from random polygons were generalized to 3-D shapes. Experiment 3 tested the role of cues to 3-D orientation (texture and shading) and the role of invariants in 3-D shape discrimination.

\section{EXPERIMENT 3 3-D Shape Discrimination}

\section{Method}

Subjects. Three subjects (the 2 authors and 1 other) were tested. The third subject (M.S.) was naive with respect to the hypotheses being tested. M.S. was a myope and used his normal glasses in the experiment. M.S.G. and Z.P. were tested in two replications of each condition, and M.S. was tested in one replication.

Stimuli. The stimuli were images of solid objects shown on a computer monitor. Two types of objects were used: elliptical cylinders and cuboids. The cylinders were viewed from a side so that none of the flat bases (top or bottom) were visible. The shape of the cylinder was represented either by texture or by shading. Texture was produced by about 100 small squares randomly positioned and oriented on the surface of the cylinder. Shading was produced by computing (using SunPhigs software) the distribution of intensity generated on the surface of a cylinder by a point light source. The light source was always in front of the cylinder, but its position randomly varied over the range of $180^{\circ}$ around the long axis of the cylinder. The height and width of the cylinder were constant (and equal to one another), but its range in depth varied randomly. In other words, the base of the cylinder was an ellipse whose one axis (orthogonal to the line of sight) had a constant length but another axis (along the line of sight) was variable. As a result, the cylinder was either flattened or elongated along the line of sight with respect to a circular cylinder, and the subject's task was to discriminate the aspect ratio. Specifically, the subject had to decide whether the aspect ratio was greater or less than one. To perform this discrimination, the subject had to reconstruct perceptually the similarity structure of the 3-D shape using a given cue (texture or shading), which provided information about local 3-D orientation of the surface.

The shape of the cuboid was represented only by the contours of the visible faces. The height and width of the cuboid were constant (and equal to one another), but its length varied randomly, so that the cuboid was either elongated or flattened, relative to a cube. As a result, two faces of the cuboid were always squares and four other faces were rectangles whose aspect ratio was changing from trial to trial. As already pointed out, a perspective, or affine, image of a square is sufficient to compute the 3-D orientation of the square (Haralick, 1989; Stevens, 1983), which in turn could be used in judgments about the shape of the cuboid. We showed in Experiment 2, however, that subjects are not likely to use an image of a square to compute the square's 3-D orientation and then to use this orientation in shape judgments. Alternatively, shape of a cuboid can be determined by using an assumption that the trihedral angles are right. In fact, it is known from prior experiments and observations that when the ob- 
server is presented with a line drawing of a cuboid (i.e., of a rectangular parallelepiped), the observer does perceive a rectangular parallelepiped. The perceptual results support the suggestion that the percept involves an assumption that the junctions in the image were produced by right trihedral angles (Attneave \& Frost, 1969; Perkins, 1972; Shepard, 1981). This assumption leads to a unique interpretation equivalent to a cuboid, and the 3-D orientation of the cuboid does not seem to be taken into account. ${ }^{10}$ This is illustrated in Figure 10, which shows an image of a cuboid. Note that this image could have been produced by an infinite number of nonrectangular parallelepipeds, but the percept seems to prefer the interpretation with right angles. This phenomenon was not studied systematically in the past. Specifically, it is not known how reliably (relative to other discrimination tasks) the assumption about right trihedral angles is used in shape perception. (Shepard, 1981, was the only one, to our knowledge, to study this problem. However, he published only qualitative results of his study showing that the subjects were able to adjust the line drawing, so that it represented, approximately, an image of a cube.)

Interestingly, the percept of a cuboid is a special case of Pizlo's (1994) theory of shape constancy based on Pizlo and Rosenfeld's (1992) algorithm. In this algorithm, shapes can be discriminated reliably when the tilts of the shapes are known (otherwise, the tilts have to be searched for; see Perspective Invariants section). But in the case of a cuboid, tilts of all faces are explicitly given in the image as directions of edges emanating from the corners of the faces (see Pizlo \& Rosenfeld, 1992, for an example of how perspective invariants can be applied to the image of a cuboid). For example, in Figure 10 , the line $a$ is a tilt direction for the face $A$. Therefore, if subjects can reliably use the information about the tilts of the faces given in the image, they should reliably discriminate among cuboids. The prior results of Attneave and Frost (1969), Perkins (1972), and Shepard (1981), as well as the observation in Figure 10, suggest that this should be the case.

Similarly, as in the case of cylinders, the subject's task was to discriminate the aspect ratio-specifically, whether the cuboid was stretched or compressed, relative to a cube. The orientation of the cuboid with respect to the observer, specified by two angles in the spherical coordinate system, was randomized: these angles varied over the range from $20^{\circ}$ to $70^{\circ}$. Note that because of randomizing the 3-D orientation of the cuboid, the subject's response could not be based on the discrimination of 2-D distances on the screen. Instead,

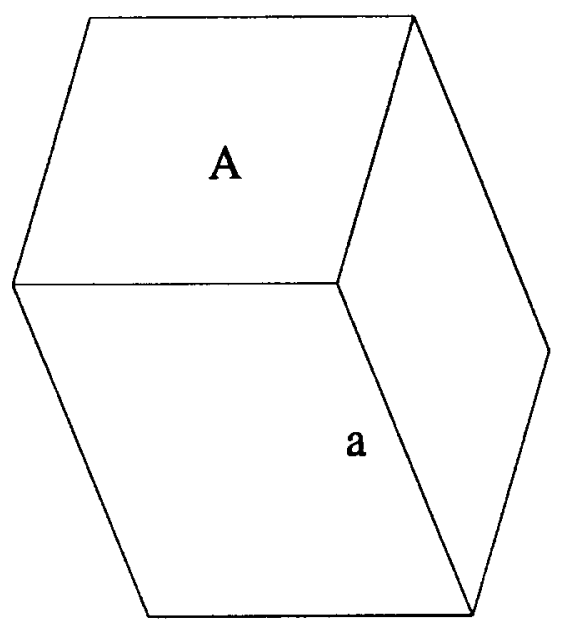

Figure 10. An image that could be produced by a rectangular parallelepiped (cuboid) and that is perceived as such. The percept is based on the assumption that images of edges (e.g., of $a$ ) are tilt directions for corresponding faces (e.g., $A$ ). the discrimination could be based only on perspective invariants (Pizlo \& Rosenfeld, 1992) specifically, on the assumption that the directions of the edges in the image are tilt directions. If the subject can use this information about the tilts, the discrimination among aspect ratio of the cuboids should be reliable.

The ratio of the range in depth of the simulated objects displayed on the monitor to the viewing distance was rather small $(0.12)$. Therefore, we produced images using orthographic projections of the objects. The exposure duration of a stimulus was $5 \mathrm{sec}$.

Procedure. The method of constant stimuli was used. There were six levels of aspect ratio (more exactly, of the logarithm of the aspect ratio) with 100 shapes per each level. Three levels of the aspect ratio were greater than one, and the other three were less than one. The range of the logarithm of the aspect ratio for each condition was established in a preliminary experiment, separately for each subject. After the set of practice trials, the subject was informed about the proportion of correct responses on trials where the aspect ratio was greater than one and on trials where the aspect ratio was less than one. All other features of the experimental method were the same as in Experiments 1 and 2 .

\section{Results and Discussion}

The relationship between the proportion of responses "aspect ratio greater than one" and the logarithm of the aspect ratio was approximated by a cumulative Gaussian distribution function using probit analysis. The quality of the approximation was quite good, as measured by a chisquare test. For Z.P., in five out of six sessions, $p>.1$, and in the remaining session, $p=.01$. For M.S.G., in five out of six sessions, $p>.1$, and in the remaining session, $p=$ .08 . For M.S. (naive subject with less practice), $p$ was in the range from .0001 to .0005 in all three sessions. The $S D$ (and its standard error) of the distribution, after converting from the logarithm of aspect ratio to the aspect ratio itself expressed in percentages, was used as an estimate of the difference threshold (and of its standard error). The difference thresholds and the standard errors of these thresholds are shown in Figure 11. It can be seen that the pattern of results is the same for all subjects. The best performance (the difference threshold from $2.6 \%$ to $6.5 \%$ ) was found for cuboids (for Z.P. and M.S.G., who received more practice in this experiment, the difference threshold was less than $5 \%$ ). Note that these thresholds are not very different from the difference threshold for line length discrimination in the frontal plane, which is about $2 \%$ when the segments to be compared had constant orientation on the retina (De Valois, Lakshminarayanan, Nygaard, Schlussel, \& Sladky, 1990; Watt, 1987) and about 5\% when the orientation of the segments randomly varied (Todd, Norman, Perotti, \& Tittle, 1993). However, in our experiment, the subjects were not simply comparing distances in the frontal plane, because these distances were not constant. Furthermore, the ratios of distances on the screen were not constant because the orientation of the cuboid was randomly changing from trial to trial. Instead, the subjects had to use a higher order variable, such as perspective invariants (Pizlo, 1994; Pizlo \& Rosenfeld, 1992).

It is worth pointing out that reliable performance that we observed for cuboids cannot be accounted for by other invariants, such as affine or projective invariants (e.g., Rothwell, Forsyth, Zisserman, \& Mundy's, 1993, algorithm for 
MSG - 1st replication

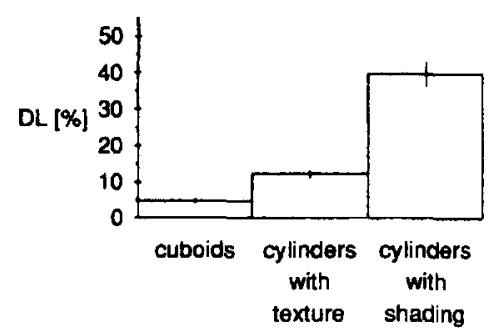

ZP - 1st replication

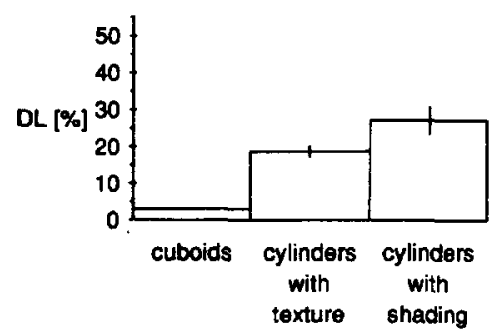

MS

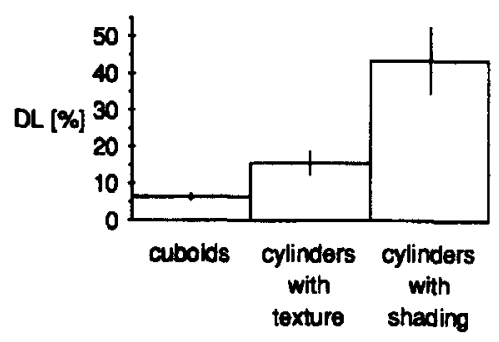

MSG - 2nd replication

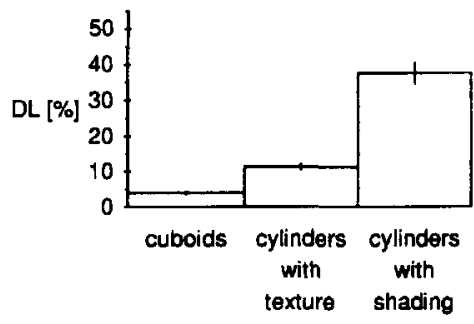

ZP - 2nd replication

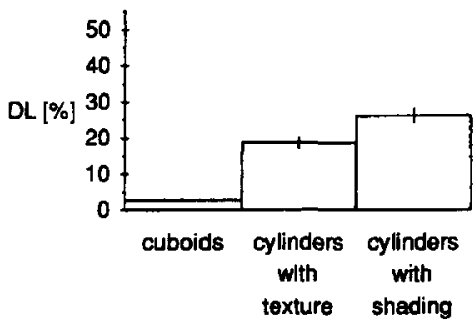

Figure 11. Difference thresholds for all 3 subjects in Experiment 3 (standard errors of the thresholds are indicated by the vertical line segments).

recognition of polyhedra by using projective invariants) because all cuboids are equivalent under affine and projective transformations and, as a result, affine and projective invariants cannot discriminate among cuboids at all.

Consider now results from cylinders. Here, perspective invariants could not be used because the stimuli did not have visible flat faces. The task could be solved only by reconstructing the Euclidean or similarity structure of the shape from texture or shading, which provide information about local 3-D orientation of the surface. It can be seen that difference thresholds are much greater for cylinders than for cuboids. For textured cylinders, the difference threshold ranged from $11 \%$ to $20 \%$; for shaded cylinders, the difference threshold ranged from $25 \%$ to $45 \%$. Note that the differences among thresholds for cuboids and for cylinders are large, relative to the standard errors of the thresholds. These results support the conclusions from Experiments 1 and 2 on flat shapes: when the object has visible flat contours, which can be used to compute perspective invariants, the discrimination is very reliable. How- ever, when the object does not have any visible flat or nearly flat contour, so that perspective invariants cannot be used and the discrimination must be based on reconstructing Euclidean or similarity structure of the object from cues to 3-D orientation of surfaces, the performance is much worse.

Consider now some limitations of Pizlo's theory and possible predictions. First, according to this theory, the percept is reliable if the object is represented by planar contours, to which perspective invariants can be applied. Consistent with this theory, for cylinders without visible flat faces, performance was poor. The question is whether this result will generalize to the shapes of tetrahedrons (polyhedrons with four triangular faces) that have flat faces, but where invariants (conventional or perspective) of faces are useless because all faces (triangles) are equivalent under perspective. ${ }^{11}$ Triangular faces could be discriminated by perspective invariants only if tilts of the faces were given. However, for tetrahedrons, tilts are not given in the image. Second, according to Pizlo's theory, if tilt directions are given explicitly, as in the case of the retinal image of a 
cuboid, the percept is based on this information. The question is, however, whether this effect of tilt directions in the retinal image is robust, so that it would still determine percept in the presence of other, conflicting cues to 3-D orientation. For example, if the picture of a cuboid is slanted relative to the observer, the directions of edges on the retina change as a function of slant, and, therefore, the assumption about right trihedral angles leads to an interpretation that depends on slant. The percept can be accurate and reliable regardless of the 3-D orientation, only if the subject can reliably use this orientation in shape perception. These two groups of predictions were tested in control experiments that are reported next.

\section{CONTROL EXPERIMENTS}

\section{Discrimination of Tetrahedrons}

Signal detection experiment was used. Two tetrahedrons were used with heights equal to $\pm 5 \%$ of the height of a regular tetrahedron (in the regular tetrahedron, all faces are equilateral triangles). For comparison, the discrimination of cuboids was repeated using two cuboids with heights equal to $\pm 5 \%$ of the height of a cube. Before the experimental sessions were run, the subject (Z.P.) received extensive practice to reduce or even remove any superiority of performance for cuboids due to the practice in the main experiment. The 3-D orientation of the objects varied randomly over the range of angles from $25^{\circ}$ to $65^{\circ}$. A smaller range of 3-D orientations was used, relative to that of the main experiment, to avoid degenerate views in the case of tetrahedron. Furthermore, the top apex of the tetrahedron was marked by a small circle to allow a unique interpretation of the stimulus. Without this mark, it would not always be clear along which direction the height was varied. Other details of the experimental method were similar to the method used in the main experiment.

Consider predictions of Helmholtzian theory and a theory based on invariants. Because the images were orthographic projections, three points are sufficient to determine the 3-D orientation of an object (Huttenlocher \& Ullman, 1988). Therefore, the 3-D orientation of the tetrahedron (or a cuboid) can be estimated first, and then the shape of the object can be discriminated by checking the position of the images of the remaining apex (apices). The alignment method would predict discrimination equally reliable for tetrahedrons as for cuboids. According to Gibsonian theory, discrimination is not possible in either case, because the objects in a given session differed only in aspect ratio. As a result, the stimuli were equivalent in affine and projective geometries and, thus, affine or projective invariants cannot be used for either cuboids or tetrahedrons. Thus, Gibsonian theory would predict similar and poor performance for both types of objects. According to Pizlo's (1994) theory, discrimination among tetrahedrons should be much worse than is that among cuboids, because the retinal image of a cuboid provides explicitly tilts of faces whereas this is not the case for tetrahedrons. Furthermore, tilts cannot even be found in tetrahedrons (whose faces are triangular) because, without knowing tilts, all triangles are perspectively equivalent. So, for cuboids, shape can be judged by using perspective invariants that allow reliable performance, but for tetrahedrons, the percept of the shape must involve integration of 3-D orientation estimated from contour and shape, as in the alignment methods.

Note that these two conditions are analogous to the Conditions 1 and 3 in Experiment 2. There, we found that integration of 3-D orientation estimated from contours of the grid and shape lowered performance by a factor of about 2.5 , relative to performance where invariants computed from the contour itself could be used. Thus, if results of Experiment 2, where we used flat shapes and texture as cues to 3-D orientation, generalize to 3-D objects with flat faces, we would expect that tetrahedrons should produce thresholds 2.5 higher than those for cuboids. Interestingly, this is exactly what was observed: threshold (corresponding to $d^{\prime}=1$ ) for cuboids was $3.1 \%$, and threshold for tetrahedrons was $7.1 \%$. These results agree with our predictions based on Pizlo's theory, and they contradict Helmholtzian and Gibsonian theories. Furthermore, the results from this control experiment suggest that the results of Experiment 2 are likely to be general and not restricted to the stimuli used in that experiment (see Results and Discussion section of Experiment 2).

Compare now the threshold for tetrahedrons with threshold for textured and shaded cylinders. These two cases are similar because in both of them shape discrimination required using a cue to 3-D orientation: the image of a contour of an equilateral triangle for tetrahedrons versus texture or shading for cylinders. It can be seen that discrimination among tetrahedrons was more reliable than was discrimination among cylinders. We believe that this difference in performance is related to the fact that the tetrahedrons, but not the cylinders, had visible flat faces. Thus, the reconstruction of the shape of a tetrahedron must involve only 3-D orientation of planar faces, whereas reconstruction of the shape of a cylinder must involve 3-D orientations as well as curvatures.

\section{Discrimination of Pictures of Cuboids}

The shape of a slanted picture of a cuboid was matched with a similar picture in the frontal plane. ${ }^{12}$ Figure 12 shows an example of a frontal view of a stimulus. According to Helmholtzian theory, the percept of the slanted picture of a cuboid should be accurate if cues to 3-D orientation are present. According to Pizlo's theory, however, the percept of a slanted picture of a cuboid should be inaccurate because the slant changes directions of images of edges on the retina. If the percept is based on the assumption that images of edges are tilts of faces, the percept of the picture of the cuboid will be inaccurate.

Note, however, that the failure of the subject in this task could be related to the difficulty in achieving shape constancy in general, rather than to the role of tilt directions on the retina as hypothesized by Pizlo (1994). To check the subject's capacity in achieving shape constancy, the subject was also tested in matching parallelograms. The parallelograms were the top faces of the pictures of the cuboids. 


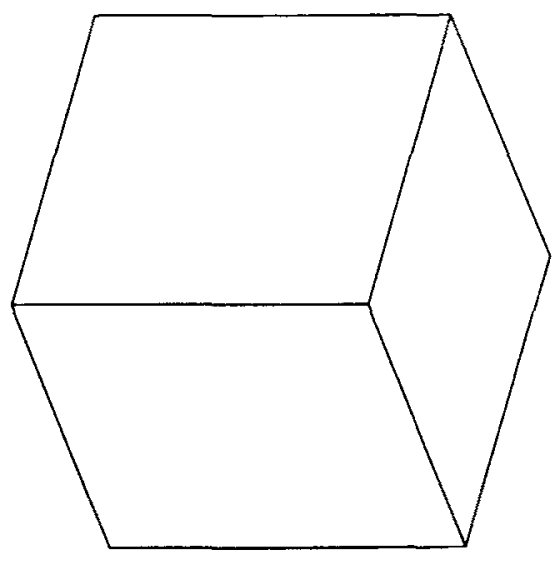

Figure 12. An example of a stimulus in a control experiment on the perception of slanted pictures of cuboids and parallelograms.

The subject's task was to adjust the aspect ratio of a cuboid or of a parallelogram (comparison shape) shown on a computer monitor to the aspect ratio of a picture of a cuboid or parallelogram (standard shape) mounted on a slanted base. The monitor was orthogonal to the line of sight of the subject's left eye. The subject's head was supported by a chin-forehead rest. The room was highly illuminated and the viewing was binocular, so that all conventional cues to 3-D orientation, other than motion, were available (the reason for excluding motion will be given after results are described). The distance of the standard shape from the subject's eye was $50 \mathrm{~cm}$, and the distance of the comparison shape from the subject's eye was $100 \mathrm{~cm}$. Five standard shapes were used in a random order. The shapes differed from one another in the aspect ratio. The pictures were always slanted with tilt $90^{\circ}$ (i.e., they were facing up and rotated around their horizontal side). Three slants were used: $50^{\circ}, 60^{\circ}$, and $70^{\circ}$. Note that the tilts and slants were not exactly the same for each eye. The values given here are for the left eye. Each picture at a slant was shown 10 times, giving rise to 150 presentations in a session (5 shapes $\times 3$ slants $\times 10$ trials). The authors served as subjects. Z.P. was tested with small shapes for which the ratio of the range in depth to the viewing distance was from .01 to .09. M.S.G. was tested with large shapes for which the ratio of the range in depth to the viewing distance was from .03 to .28 .

The subject's performance was evaluated by comparing the aspect ratio of the comparison (adjusted) shape with the aspect ratio of the standard shape. The results are given after normalizing the adjusted aspect ratio of the comparison shape to the aspect ratio of the standard shape. So, accurate judgment is represented by 1.00 . On the average, the retinal aspect ratio was .5 of the aspect ratio of the standard shape. Thus, the complete failure of shape constancy would be represented by average judgment of 0.50 .

Mean adjusted shape for parallelograms was 0.93 and 1.00 for Z.P. and M.S.G., respectively, whereas mean adjusted shape for the cuboids was 0.72 and 0.86 for Z.P. and M.S.G., respectively. Standard error of each of these esti- mates was not larger than 0.01 . These results show that the performance with the pictures of cuboids was substantially less accurate than was performance with the pictures of parallelograms. If the subjects were reconstructing the Euclidean or similarity structure of the shapes after taking the 3-D orientation into account, they should be equally or even more accurate with pictures of cuboids because these pictures contained three parallelograms that had the same 3-D orientation (hence, the subjects were presented with redundant information about the 3-D orientation). However, the performance was worse with cuboids. This suggests that the percept was affected by the retinal tilt directions, as predicted by Pizlo's (1994) theory. The reader is encouraged to view Figure 12 from different viewing directions to observe the effect of slant on the percept. If the axis of rotation is horizontal, the cuboid appears compressed, relative to a cube. If the axis of rotation is vertical, the cuboid appears stretched. Note further that M.S.G. produced more accurate judgments than did Z.P. This can be related to the fact that M.S.G. was presented with larger stimuli that gave rise to larger perspective effects. Note that perspective, rather than affine, effects allowed the subjects to discriminate among the stimuli, because the stimuli were equivalent under affine transformation (recall that the stimuli differed from one another by aspect ratio).

Next, we want to compare our results with those of a recent study by Todd and Norman (1994). They showed that, in the presence of all cues to 3-D orientation and distance, subjects were not able to judge distances reliably or accurately. In our control experiment, however, we found that the subjects were quite accurate and precise in judging aspect ratio of parallelograms. This difference is likely to be related to the fact that Todd and Norman's subjects were required to judge distances, which are Euclidean properties, whereas our subjects were required to judge the aspect ratio of a planar contour, which is a perspective property. Thus, if subjects have a reliable perceptual access to perspective properties of stimuli (as described by Pizlo's theory), but not to Euclidean properties, they can reliably and accurately solve our task, but not Todd and Norman's task. It is important to point out that although the set of standard distances in Todd and Norman's study formed a planar polygonal pattern, the comparison stimulus was just a line segment. In our control experiment, both the standard and the comparison stimuli were planar shapes. Thus, the difference was essentially related to the type of the comparison stimulus (line segment in Todd and Norman's experiment, and planar shape in ours). Note that Pizlo and Rosenfeld's algorithm can be used only if two planar shapes are to be compared. The fact that the human observers were accurate and reliable in our task but not in Todd and Norman's task provides further support for Pizlo's theory.

Finally, note that the fact that the percept of the slanted picture of a cuboid is not veridical and that the percept is determined by tilt directions on the retina suggests that, if the picture performs a planar rotation, the picture may look nonrigid. This is exactly what happens, as was shown by Pizlo and Salach-Golyska (1994b) (again, the 
reader is encouraged to use Figure 12 to observe this effect). This nonrigidity was a reason why the subjects in this control experiment had the head supported by a chinforehead rest. Otherwise, it would be difficult to adjust the comparison shape to the standard one. This observation suggests that motion is not a reliable cue to 3-D orientation. This suggestion receives some support from a recent study by Durgin et al. (1994). There are, however, studies suggesting exactly the opposite - namely, that motion is a very efficient cue that allows reconstructing similarity or metric structure of an object (Johnston et al., 1994; Lappin \& Love, 1992; but see also Lappin \& Ahlstrom, 1994 and Pizlo \& Salach-Golyska, 1994a). To shed more light on the effectiveness of motion in judgments about similarity structure on a slanted plane, relative to other types of information used in Experiments 1-3 (perspective invariants, texture, shading, binocular vision), we performed Experiment 4 with the same subjects (the authors) and similar stimuli as in the other three experiments.

\section{EXPERIMENT 4 \\ Distance Discrimination From Planar Motion}

\section{Method}

Subjects. The authors served as subjects. Z.P. was tested in three sessions, and M.S.G. was tested in one session.

Stimuli. On each trial, two random polygons were shown side by side. The polygons performed two cycles of rigid planar motion $\left( \pm 45^{\circ}\right)$ around a random point between the polygons on a slanted plane. The slant varied randomly from trial to trial over the range $40^{\circ}-60^{\circ}$. Tilt was always $90^{\circ}$ (the plane faced up). The duration of each trial was about $2 \mathrm{sec}$. The polygons were generated using the algorithm described in the Method section of Experiment 1. Two points were then randomly chosen on the circumference of each polygon. These two pairs of points specified distances on a slanted plane that were to be discriminated.

Procedure. The method of constant stimuli was used. There were six levels of the ratio of the two distances to be discriminated and 100 trials per each level were used. The subject was asked to decide whether the distance on the right side was greater or less than the one on the left side.

All other features of the experimental method were the same as those in Experiments 1-3.

\section{Results and Discussion}

The relation between the proportion of responses "greater" and the ratio of the two distances was approximated by a cumulative Gaussian distribution function using probit analysis. The fit was good, as measured by a chi-square test ( $p>.15$ ). The $S D$ (and its standard error) of the distribution was used as the estimate of the threshold and its standard error. Difference thresholds were in the range $9.8 \%-11.7 \%$ (standard error of the difference threshold was about $0.8 \%$ ). These thresholds are similar to the thresholds from texture condition in Experiment 3, and they are much higher than thresholds estimated for cuboids. This suggests that, like texture, contour, or shading, planar motion does not lead to reliable perception of Euclidean or similarity structure based on taking 3-D orientation into account. This fact provides support for the explanation of the cuboid illusion originally described by the authors (Pizlo \& Salach-Golyska, 1994b): namely, the percept is reliable when the shape of a planar contour is judged and the tilt of the contour is explicitly given, but the percept is unreliable otherwise. When planar contours and tilt directions provide inaccurate information (as they do for the slanted picture of a cuboid), and this information is in conflict with cues to 3-D orientation, the 3-D orientation may be ignored and, as a result, the percept may be inaccurate. We want to point out, however, that before the hypothesis about planar motion as a reliable source of perceptual information about metric structure on a plane is rejected, further experiments are needed. Such experiments should test the nature of information (e.g., Euclidean vs. projective) provided to the observer by planar motion, as well as the extent to which the observer uses this information in space and shape perception. Such experiments, however, are beyond the scope of this paper.

To conclude, the results presented in this paper show that shape perception is based on invariants computed from images of flat contours, and cues to 3-D orientation do not play an important role. Obviously, this does not mean that cues to 3-D orientation cannot be used in shape perception. They can, but performance based on such cues is less reliable (see Experiment 3 ). As a result, if invariants and cues to 3-D orientation provide consistent information about the shape of an object, the subject's performance is not based on integrating or fusing the two types of information so that the percept could be even more reliable. Instead, it is exclusively based on invariants (as in our Experiment 2 and in Stavrianos's experiment). This perceptual dominance of invariants over cues to 3-D orientation has an interesting implication. Namely, if invariants are in conflict with cues to 3-D orientation, the percept is still likely to be determined by invariants, even though the percept may not be veridical. This can produce illusions. Cube illusion, described above, is one example (Pizlo \& SalachGolyska, 1994b). Another example was provided by Johnston, Cumming, and Parker (1993), where they showed images of cubes with correct versus incorrect texture. If the percept was based on texture as a cue to 3-D orientation of surfaces, the perceived shape would be very different from shape determined by contour. The observation of their Figure 3 does not seem to illustrate any effect of texture on the percept. Unfortunately, Johnston et al. (1993) did not test the effect of planar contours and they did not use cubes as stimuli. Results of our experiments, as well as Pizlo's (1994) theory and the observation of Johnston et al.'s (1993) Figure 3, predict no, or very little, effect of texture, if perspective invariants computed from planar contours can be used.

In the next section, we remind the reader about two other illusory percepts produced by the conflict of contours and conventional cues to 3-D orientation that were described, but not explained, previously. We will conjecture that these illusions are observed because the percept relies on invariants computed from contours, rather than on more adequate cues to $3-\mathrm{D}$ orientation. 


\section{IMPLICATIONS FOR INTEGRATING INVARIANTS AND DEPTH CUES}

Consider first the illusion similar to that presented by Cavanagh and Grunau (1989). Figure 13 shows a drawing of a human face that is neither sad nor smiling. Look what happens when you add the third dimension to this object by folding the page in a U-shape way (the edges of the mouth should be at distance from the observer different from the center of the mouth). Note that after folding the page, the contours representing the face, particularly the mouth, are not flat. As a result, the perceptual mechanism based on perspective invariants, which operates on images of contours assuming that the contours are flat, cannot lead to accurate percept. This problem could be compensated if cues to 3-D orientation (or curvature) were effectively used. However, the cues to 3-D orientation are not used. Slowly rotate the page around the horizontal axis. In one position, the face is perceived as sad; in the other position, it is perceived as smiling. This illustration shows that having all cues to 3-D orientation available, as well as being perfectly aware of the way this object was made, you are not able to use this knowledge to reconstruct the actual Euclidean (or similarity) structure of this 3-D face. The percept is based on the image of the contours, and the illusion arises because the contours are not flat.

Next, consider the illusion described by Ramachandran (1990). The illusion involves the structure-from-motion phenomenon described by Ullman (1979) and related to the kinetic-depth effect (Wallach \& O'Connell, 1953). If a number of dots are moving in a display as if they were placed on the surface of a rotating cylinder, the observer perceives a rotating cylinder. Note that, in this case, the

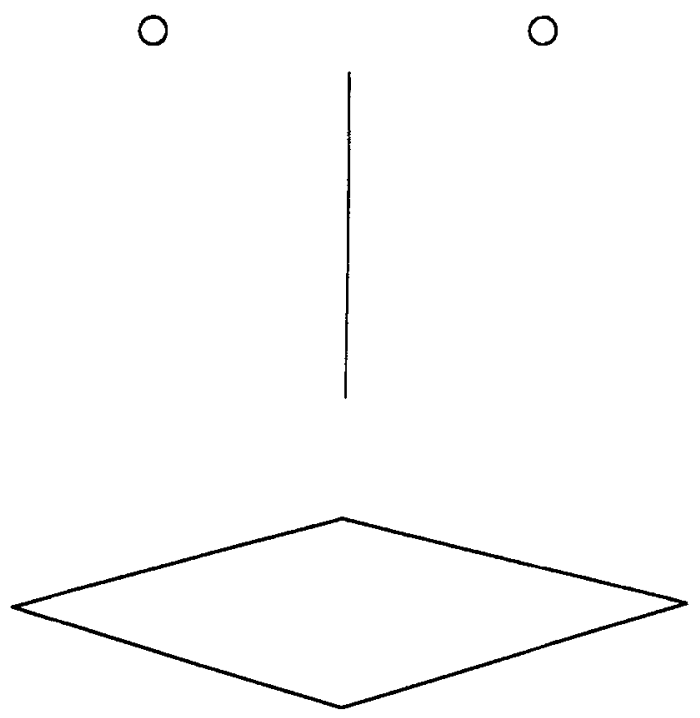

Figure 13. Curl this page so that you are looking into a $U$ (i.e., form a smooth curve about the long axis of this page); then, slowly rotate the page around its horizontal axis. When the top of the page is near, the face is perceived as sad. When the bottom of the page is near, the face is perceived as smiling (after Cavanagh $\&$ Grunau, 1989). cue (motion) to 3-D orientation (or curvature) and the flat (occluding) contour do not contradict each other. Now, if the observer is looking at the same stimulus, but through a circular aperture, then the observer perceives a rotating sphere. If the aperture is triangular, then a rotating cone is perceived. Note that, in the two latter cases, the cue to the 3-D orientation and curvature and the flat (occluding) contour were inconsistent. Interestingly, the percept was determined primarily by the contour and motion was responsible only for the qualitative impression of the third dimension.

\section{REFERENCES}

Attneave, F., \& Frost, R. (1969). The determination of perceived tridimensional orientation by minimum criteria. Perception \& Psychophysics, 6, 391-396.

BAJCSY, R. (1985). Active perception vs. passive perception. In Workshop on computer vision: Representation and control (pp. 55-59). Washington, DC: IEEE Computer Society Press.

BECK, J., \& Gibson, J. J. (1955). The relation of apparent shape to apparent slant in the perception of objects. Journal of Experimental Psychology, 50, 125-133.

CASSiRER, E. (1944). The concept of group and the theory of perception. Philosophy \& Phenomenological Research, 5, 1-35. (Original work published 1938)

CAVANAGH, P., \& GRÜNAU, M. voN (1989). 3-D objects that appear nonrigid during rotation. Investigative Ophthalmology \& Visual Science, 30 (Suppl). 263.

Courant, R., \& RobBins, H. (1941), What is mathematics? New York Oxford University Press

De Valois, K. K., Lakshminarayanan, V., Nygaard, R., SchlusSEL, S., \& SLADKY, J. (1990). Discrimination of relative spatial position. Vision Research, 30, 1649-1660.

Durgin, F. H., ProffitT, D. R., Olson, T. J., \& Reinke, K. S. (in press) Comparing depth from binocular disparity to depth from motion. Journal of Experimental Psychology: Human Perception \& Performance.

Edelman, S., \& BÜLTHOFF, H. H. (1992). Orientation dependence in the recognition of familiar and novel views of three-dimensional objects. Vision Research, 32, 2385-2400.

GiBson, J. J. (1950). The perception of the visual world. Boston: Houghton Mifflin.

Gibson, J. J. (1979). The ecological approach to visual perception. Boston: Houghton Mifflin.

HARALICK, R. M. (1989). Determining camera parameters from the perspective projection of a rectangle. Pattern Recognition, 22, 225-230.

HoNG, J., \& TAN, X. (1989). Recognize the similarity between shapes under affine transformation. In Proceedings of the International Conference on Computer Vision (pp. 489-493). New York: IEEE Computer Society Press.

HUTtENLOCHER, D. P., \& Ullman, S. (1988). Recognizing solid objects by alignment. In Proceedings of DARPA Image Understanding Workshop (pp. 1114-1124). San Mateo, CA: Morgan Kaufmann.

JoHANSSON, G. (1977). Spatial constancy and motion in visual perception. In W. Epstein (Ed.), Stability and constancy in visual perception: Mechanisms and processes (pp. 375-419). New York: Wiley.

Johnston, E. B., Cumming, B. G., \& LANDY, M. S. (1994). Integration of stereopsis and motion depth cues. Vision Research, 34, 2259-2275.

Johnston, E. B., Cumming, B. G., \& PARKer, A. J. (1993). Integration of depth modules: Stereopsis and texture. Vision Research, 33, 813-826.

KAISER, P. K. (1967). Perceived shape and its dependency on perceived slant. Journal of Experimental Psychology, 75, 345-353.

LAPPIN, J. S., \& AHLSTRÖM, U. B. (1994). On the scaling of visual space from motion-in response to Pizlo and Salach-Golyska. Perception \& Psychophysics, 55, 235-242.

LAPPIN, J. S., \& LovE, S. R. (1992). Planar motion permits perception of metric structure in stereopsis. Perception \& Psychophysics, 51, 86-102. LARSEN, A. (1985). Pattern matching: Effects of size ratio, angular dif- 
ference in orientation, and familiarity. Perception \& Psychophysics, 38, 63-68.

Leibowitz, H. W., Wilcox, S. B., \& Post, R. B. (1978). The effect of refractive error on size constancy and shape constancy. Perception, 7 , 557.562 .

LowE, D. G. (1987). Three-dimensional object recognition from single two-dimensional images. Artificial Intelligence, 31, 355-395.

LUCE, R. D. (1986). Response times. New York: Oxford University Press. Luce, R. D., \& Galanter, E. (1963). Discrimination. In R. D. Luce, R. R. Bush, \& E. Galanter (Eds.), Handbook of mathematical psychology (Vol. 1, pp. 191-243). New York: Wiley.

MARR, D. (1982). Vision. New York: W. H. Freeman.

Mundy, J. L., \& ZisSERMAN, A. (1992). Geometric invariance in computer vision. Cambridge, MA: MIT Press.

PERKINS, D. N. (1972). Visual discrimination between rectangular and nonrectangular parallelopipeds. Perception \& Psychophysics, 12, 396-400.

Pirenne, M. H. (1975). Vision and art. In E. C. Carterette \& M. P. Friedman (Eds.), Handbook of perception: Seeing (pp. 443-490). New York: Academic Press.

Pizlo, Z. (1988). Physiology based simulation model of triangle shape recognition. Biological Cybernetics, 58, 51-62.

PizLo, Z. (1993). Geometry of visual space. Investigative Ophthalmology \& Visual Science, 34, 708. (Abstract)

PizLo, Z. (1994). A theory of shape constancy based on perspective invariants. Vision Research, 34, 1637-1658.

Pizlo, Z., \& Rosenfeld, A. (1992). Recognition of planar shapes from perspective images using contour-based invariants. CVGIP: Image Understanding, 56, 330-350.

Pizlo, Z., Rosenfeld, A., \& Weiss, I. (in press-a). The geometry of visual space: About the incompatibility between science and mathematics. Computer Vision \& Image Understanding.

Pizlo, Z., Rosenfeld, A., \& Weiss, I. (in press-b). Interdisciplinary study of visual invariants. In D. Dori \& A. Bruckstein (Eds.), Structure and syntax in pattern recognition. Singapore: World Scientific.

Pizlo, Z., \& SALACH-GolySKA, M. (1994a). Is vision metric? Comment on Lappin and Love (1992). Perception \& Psychophysics, 55, 230-234.

Pizlo, Z., \& SAlach-GolysKa, M. (1994b). Monocular, binocular and multiview perception. Investigative Ophthalmology \& Visual Science, 35, 1916. (Abstract)

PizLo, Z., \& SCHEESSELE, M. R. (1995). On some geometrical aspects of shape perception. Manuscript submitted for publication.

Poggio, T., Torre, V., \& Koch, C. (1985). Computational vision and regularization theory. Nature, 317, 314-319.

RAMACHANDRAN, V. S. (1990). Visual perception in people and machines In A. Blake \& T. Troscianko (Eds.), AI and the eye (pp. 21-77). New York: Wiley.

Rock, I., \& DiViTA, J. (1987). A case of viewer-centered object perception. Cognitive Psychology, 19, 280-293.

Rock, I., WheELER, D., \& TUdoR, L. (1989). Can we imagine how objects look from other viewpoints? Cognitive Psychology, 21, 185-210.

Rothwell, C. A., Forsyth, D. A., Zisserman, A., \& Mundy, J. L. (1993). Extracting projective structure from single perspective views of $3 D$ point sets. In Proceedings of the IEEE International Conference on Computer Vision (pp. 573-582). Los Alamitos, CA: IEEE Computer Society Press.

SHEPARD, R. N. (1981). Psychophysical complementarity. In M. Kubovy \& J. R. Pomerantz (Eds.), Perceptual organization (pp. 279-341). Hillsdale, NJ: Erlbaum.

SPRINGER, C. E. (1964). Geometry and analysis of projective spaces. San Francisco: W. H. Freeman.

Stavrianos, B. K. (1945). The relation of shape perception to explicit judgments of inclination. Archives of Psychology, 296, 1-94.

STEVENS, K. A. (1983). Surface tilt (the direction of slant): A neglected psychophysical variable. Perception \& Psychophysics, 33, 241-250.

Thouless, R. H. (1931). Phenomenal regression to the real object II. British Journal of Psychology, 22, 1-30.

Thouless, R. H. (1934). The general principle underlying effects attributed to the so-called phenomenal constancy tendency. Psychologische Forschung, 19, 300-310.

ToDD, J. T., \& Bressan, P. (1990). The perception of 3-dimensional affine structure from minimal apparent motion sequences. Perception \& Psychophysics, 48, 419-430.
TodD, J. T., \& Norman, J. F. (1991). The visual perception of smoothly curved surfaces from minimal apparent motion sequences. Perception \& Psychophysics, 50, 509-523.

TodD, J. T., \& Norman, J. F. (1994). The visual perception of 3D length in natural vision. Investigative Ophthalmology \& Visual Science, 35 , 1329. (Abstract)

Todn, J. T., Norman, J. F., Perotri, V. J., \& Tittle, J. T. (1993). The discrimination of $3 \mathrm{D}$ length from motion and stereopsis. Investigative Ophthalmology \& Visual Science, 34, 1131. (Abstract)

TODD, J. T., \& REICHEL, F. D. (1989). Ordinal structure in the visual perception and cognition of smoothly curved shapes. Psychological Review, 96, 643-657.

UlLman, S. (1979). The interpretation of visual motion. Cambridge, MA: MIT Press.

Wallach, H., \& O'Connell, D. N. (1953). The kinetic depth effect. Journal of Experimental Psychology, 45, 205-217.

WATT, R. J. (1987). Scanning from coarse to fine spatial scales in the human visual system after the onset of a stimulus. Journal of the Optical Society of America A, 4, 2006-2021.

WITKIN, A. P. (1981). Recovering surface shape and orientation from texture. Artificial Intelligence, 17, 17-45.

Wolfe, W. J., Mathis, D., Sklair, C. W., \& Magee, M. (1991). The perspective view of three points. IEEE Transactions on Pattern Analysis \& Machine Intelligence, 13, 66-73.

\section{NOTES}

1. Tilt specifies the 2-D orientation of the axis of the 3-D rotation. More exactly, tilt is the orientation of the projected image of a normal to the surface. Slant specifies the amount of rotation in depth. More exactly, slant is the angle between the line of sight and the normal to the surface. According to this definition, the frontal plane has a slant zero.

2. It has to be pointed out, however, that despite the fact that Equations 2 do not have a natural visual interpretation (because, in a visual system, the image on the retina is never transformed to the object), the analysis of Equations 2 can be used to explain visual perception. Equations 1 can be used to determine the family of retinal images produced by a single object under different orientations relative to the observer, whereas Equations 2 can be used to determine the family of objects that, under different orientations relative to the observer, produce a given image on the retina. As a result, invariants derived from Equations 1 will allow verifying whether a given retinal image belongs to the class of images produced by a given object, and invariants derived from Equations 2 will allow verifying whether a given object belongs to the class of objects that produce a given retinal image. Clearly, either of these approaches can be used to solve the shape constancy problem, and, therefore, the choice will be based on computational efficiency.

3. Interestingly, Johansson (1977) seemed to have some intuition of this kind of approach. First, he postulated, after Cassirer (1938/1944), Courant and Robbins (1941), and Gibson (1950), that vision may require a model that is different from a Euclidean geometry - most likely, projective geometry. Then, he pointed out that projective relations themselves are not sufficient to explain visual perception. Specifically, he used the vector space model, which involved properties of perspectivity, not projectivity. For example, if the first image is a large square, and the second image (shown after the first disappears) is a smaller rectangle, the observer perceives an object moving in depth and changing shape. Johansson's explanation of this percept is based on the assumption that perceptually a square and a rectangle are not equivalent. But this assumption contradicts the projective and affine geometries as models of vision, because, in projective and affine geometries, any two rectangles (including square as a special case of a rectangle) are equivalent. As a result, if the percept involved projective or affine geometry, no change of shape would be observed. Johansson postulated that projective geometry is a good starting point, which then has to be supplemented by something else (e.g., vector spaces). All these remarks and assumptions seem to be pointing toward the kind of claims that we make. However, Johansson never explained or explored the differences between projective or affine and perspective.

4. This example also illustrates limitations of affine geometry as a model of human vision. In affine geometry, a trapezoid can never be an image of a square and, therefore, according to affine geometry, a trap- 
ezoid on the retina cannot lead to the percept of a square. In human vision, however, a square can give rise to a trapezoid on the retina, and this retinal image can be interpreted as a square (Beck \& Gibson, 1955).

5. The assumption that the standard shape is known to the observer seems to be reasonable in natural vision, where recognition of shapes (or shape constancy phenomenon) involves familiar objects that were likely to be seen by the observer when they were in the observer's frontal plane. In fact, most, if not all, shape constancy experiments used a standard shape in the frontal plane (e.g., Stavrianos, 1945). This assumption, however, does not have to be satisfied if image formation can be approximated by an affine transformation (i.e., when the range of the objects in depth is small relative to the distance of the objects from the observer). Justification of this claim will be given after the computational method of shape recognition is described.

6. Note, however, that since image formation has three parameters, the composition of two image formations has at most six parameters. But general projectivity has eight parameters. This means that two retinal images are related by a transformation, which is a subset of projective transformations, rather than a general projective transformation. Mathematical properties of this subset have not yet been analyzed, however, and the psychological relevance of these transformations is open for future research.

7. The fact that perspective stimuli used in this experiment were very similar to affine implies that this experiment did not test whether shape perception follows the rules of perspective as opposed to affine. It is almost obvious from our everyday life experience, however, that our shape and space perception does involve properties of perspective and that properties of affine transformation are not general enough (see Note 4). These phenomenoiogical observations were tested experimentally by Beck and Gibson (1955) and Kaiser (1967). Beck and Gibson showed that, for a given retinal image, the subject's percept corresponds more often to a shape that could produce this image under perspective (more specifically, under image formation) than to other shapes. Kaiser (1967) studied this problem in more detail, and his results show that a given retinal image (trapezoid) gave rise to percepts from the family of perspectively equivalent trapezoids (more exactly, trapezoids equivalent under inverse image formation as represented by Equations 2). This can be verified by the analysis of his results shown in his Table 1. From the perceived ratio of the top base to the bottom base of the trapezoid in his experiment, one can predict the perceived aspect ratio (height-to-base ratio) by applying the rules of perspective in one case or the rules of affine in another. The axis of rotation was horizontal, and it coincided with the bottom base of the trapezoid. According to affine transformation, the aspect ratio of the perceived trapezoid should not be related to the perceived ratio of the bases; however, according to perspective transformation, it should. Inspection of the results in his Table 1 shows that perceptually the aspect ratio was related to the ratio of the bases. To verify this observation quantitatively, we computed the correlation between the perceived aspect ratio of the trapezoid (using Kaiser's data from his Table 1, for monocular viewing) and predicted aspect ratio by applying the rules of image formation to the perceived ratio of the two bases of the trapezoid. This correlation was $0.90(p<.001)$. This highly significant correlation suggests that the percept follows the rules of perspective, rather than the rules of affine (in the case of affine this correlation should be zero). On the average, the perceived aspect ratio was $10 \%$ smaller than the predicted aspect ratio, which represents systematic $(p<.01)$ but small departure of subjective perspective from geometrical perspective in the direction of exaggerated perspective.

8. We modified the measure of the nonperspective differences used in Pizlo and Rosenfeld's (1992) algorithm. In their original algorithm, the nonperspective differences were measured by $S D$ of the histogram of $\rho$ (see Perspective Invariants of Shape section). However, as Tom Shepherd suggested (personal communication, August 27, 1991), mean deviation ( $m d e v$ ) of the histogram is a better measure of the nonperspective differences than is $S D$ (mean deviation is an average of absolute deviations around the mean), because mdev is less sensitive to outliers produced by small changes of local properties of a shape. Next, note that the value of $m d e v$ (or $S D$ ) depends to some extent on the position of the point on the contour that is used as the starting point in both $\Psi$ functions. To eliminate this additional source of variability, we used the average of mdevs computed for starting points at all vertices of the polygons (for simplicity of notation the average of mdevs will be denoted by $m d e v$ in the rest of this paper). In the computations, we assumed that the subject knew the correspondence among the vertices of the standard and comparison shapes. This assumption seems reasonable because the computation of the comparison shape did not involve rotation of the shape on the screen. As a result, the subjects could easily establish this correspondence, at least for comparison shapes that were perspective or close to perspective images of the standard (see Figure 7). If the comparison shape was very different from perspective image, the "correct" correspondence did not really exist and, as a result, the correspondence used by the algorithm and by the observer could be different. This could increase the variability of the data points, but it should not cause any serious problem because, in such a case, the value of mdev was likely to be large for any correspondence.

9. The statistically significant heterogeneities $(p<.1)$ that we observed in four cases were probably produced by the variability in the stimulus, which was not under the experimenter's control. First, note that each data point represented a range of $\log (m d e v)$, rather than a single value of $\log (m d e v)$. Second, the value of $\log (m d e v)$ might not be accurate because the correspondence among the vertices of the standard and comparison shapes established by the subject could be different from the correspondence used by the algorithm. In such a case, the perceived amount of the nonperspective differences could be different from the amount measured by the algorithm (see Note 8 ).

10. It has to be pointed out that sometimes a given junction in the image cannot be an image of a right trihedral angle because of geometrical constraints. In such cases, the subjects quite reliably discriminate between junctions that could or could not be produced by a right trihedral angle (Perkins, 1972). However, there has been no study on the perceived shape of a parallelepiped, when its image cannot be interpreted as a cuboid.

11. We are grateful to James Todd, who suggested this control experiment.

12. This control experiment is relevant to the problem of viewing pictures from a side (e.g., Pirenne, 1975), but the discussion of this problem is beyond the scope of this paper, and it will be presented elsewhere (Pizlo \& Scheessele, 1995).

(Manuscript received February 7, 1994; revision accepted for publication December 20, 1994.) 